\title{
GEOMETRIC HAMILTONIAN STRUCTURES ON FLAT SEMISIMPLE HOMOGENEOUS MANIFOLDS*
}

\author{
G. MARÍ BEFFA ${ }^{\dagger}$
}

\begin{abstract}
In this paper we describe Poisson structures defined on the space of Serret-Frenet equations of curves in a flat homogeneous space $G / H$ where $G$ is semisimple. These structures are defined via Poisson reduction from Poisson brackets on $\mathcal{L} \mathfrak{g}^{*}$, the space of Loops in $\mathfrak{g}^{*}$. We also give conditions on invariant geometric evolution of curves in $G / H$ which guarantee that the evolution induced on the differential invariants is Hamiltonian with respect to the most relevant of the Poisson brackets. Along the way we prove that differential invariants of curves in semisimple flat homogeneous spaces have order equal to 2 or higher, and we also establish the relationship between classical moving frames (a curve in the frame bundle) and group theoretical moving frames (equivariant $G$-valued maps on the jet space).
\end{abstract}

Key words. Invariant evolutions of curves, flat homogeneous spaces, Poisson brackets, differential invariants, completely integrable PDEs, moving frames

AMS subject classifications. Primary 37K; Secondary 53A55

1. Introduction. The subject of infinite dimensional Hamiltonian structures has applications to different branches in mathematics but its study has traditionally been an important component in the study of completely integrable systems. In fact, the majority of completely integrable systems of PDEs are Hamiltonian with respect to two different but compatible infinite dimensional Hamiltonian structures, that is, they are biHamiltonian. This property allows the generation of a recursion operator that produces an infinite sequence of preserved functionals.

The connection between classical differential geometry and completely integrable PDEs dates back to Liouville, Bianchi and Darboux ([Li], [Bi], [Da]), but it was after Hasimoto's work in the vortex filament flow evolution that the close relation between integrable PDEs and the evolution of curvature and torsion (rather than the curve flow itself) was clear. In fact, Hasimoto ([Ha]) proved that the vortex filament flow induces a completely integrable evolution on the curvature and torsion of the flow. In particular, the evolution of curvature and torsion was biHamiltonian. Langer and Perline pointed out in their papers on the subject (see [LP1], [LP2]) that the Hamiltonian structures that were used to integrate some of these systems were defined directly from the Euclidean geometry of the flow. This situation was known to exist not only in Riemannian geometry but also in projective geometry. In fact, the Schwarzian KdV equation

$$
u_{t}=u_{x} S(u)
$$

where $S(u)=\frac{u_{x x x} u_{x}-3 / 2 u_{x x}^{2}}{u_{x}^{2}}$ is the Schwarzian derivative of $u$, has been known to be a curve evolution inducing a KdV evolution on $S(u)$. The Schwarzian derivative $S(u)$ is the differential invariant of reparametrizations of the projective line (or curves in $\left.\mathbb{R P}^{1}\right)$. There are also $\operatorname{PSL}(n, \mathbb{R})$-invariant flows of curves in $\mathbb{R P}^{n}$ inducing generalized KdV equations on the projective differential invariants of the flow ([DS], [M2]). The Hamiltonian structures used to integrate them can be defined directly from the

\footnotetext{
*Received November 14, 2006; accepted for publication March 22, 2007.

${ }^{\dagger}$ Mathematics Department, University of Wisconsin, Madison, Wisconsin 53706, USA (maribeff@ bmath.wisc.edu).
} 
projective geometry of the curves ([M2]). Still, the methods used in [M2] are complicated and non intuitive. Thus, the question of integrability of invariant geometric evolutions can be shifted to a question of integrability of the flow of the differential invariants along the curve and to the study of compatible Hamiltonian structures on the space of invariants and whether or not they can be obtained from the local geometry.

Although integrable systems is a motivation for this paper, it is not its subject. In this paper we describe the general procedure that allows the generation of Poisson brackets from the geometry of curves in a flat semisimple homogeneous space. Furthermore, we give conditions on geometric flows guaranteeing that the flow of their invariants is Hamiltonian with respect to the new found brackets. Semisimplicity is needed for the definition of the bracket, although some nonsemisimple cases (e.g. the semidirect product of a semisimple group with $\mathbb{R}^{n}$ ) can be reduced to the semisimple situation (see [M1] for the Euclidean case and [M4] for the general one). Also, being flat is not necessary for much of the study, but except in the three dimensional constant curvature Riemannian case, the general role of the curvature in the Poisson geometry of differential invariants is still unknown, so we will restrict ourselves to the flat case.

The procedure can be described as follows: Assume our manifold is a flat homogenous space of the form $G / H$, where $G$ is semisimple and its Lie algebra is $|1|$-graded. In section 5 we first choose an appropriate moving frame along curves using Fels and Olver's moving frame method ([FO1], [FO2]). A key result to the paper is to prove that the space of Serret-Frenet equations (or differential invariants) $\mathcal{K}$ can be identified with a quotient of a submanifold of the (regular part) of the dual of the algebra of Loops in the Lie algebra, $\mathcal{L}_{\mathfrak{g}}$. In fact, we can find a submanifold $U \subset \mathcal{L} \mathfrak{g}^{*}$ and a foliation $\Phi$ such that $\mathcal{K} \cong U / \Phi$. The result is described in Theorem 6 . There is a well-known Poisson bracket defined on $\mathcal{L} \mathfrak{g}^{*}$. The bracket is induced on $\mathcal{L} \mathfrak{g}^{*}$ by the Lie-Poisson bracket of the Kac-Moody algebra of $\mathfrak{g}$ associated to $S^{1}$. We show that this Poisson bracket is Poisson reducible to the quotient $U / \Phi$, and hence it defines a Poisson bracket on the manifold of differential invariants (Theorem 7). Another family of simpler and compatible brackets on $\mathcal{L}^{*}$ are defined in section 7 , one can then investigate their reductions in a case-by-case basis. When reducible, these brackets are all compatible and they induce compatible Hamiltonian structures on the space of differential invariants. All best known Poisson brackets used in the integration of PDEs can be obtained in this geometric way. Except for the planar case ([CQ1], [CQ2]), integrable systems associated to conformal and other geometries are largely unexplored. Their study is likely to shed light on the geometry of curves itself.

Once we identify Poisson structures on the space of differential invariants, we would like to know which invariant geometric evolutions of curves will induce a Hamiltonian evolution on the differential invariants. Evolutions of curves which are invariant under the group action are known to be of the form

$$
u_{t}=R \mathbf{r}
$$

where $R$ is a matrix defined by a classical moving frame (in invariant theory $R$ is an invertible matrix of vector relative differential invariants associated to a Jacobian weight) and where $\mathbf{r}$ is a vector of differential invariants. In section 6 , Theorem 10, we show that if we can find a Hamiltonian functional $h: \mathcal{K} \rightarrow \mathbb{R}$ defined on the space of differential invariants $\mathcal{K}$ and an extension $\mathcal{H}: \mathcal{L}^{*} \rightarrow \mathbb{R}$ constant on the leaves of $\Phi$ and such that the component of $\frac{\delta \mathcal{H}}{\delta L}$ in the direction of $T_{e}(G / H)$ coincides with $\mathbf{r}$, 
then the evolution induced on the differential invariants by the $u$-evolution above is Hamiltonian with respect to the Kac-Moody reduced bracket and $h$ is its associated Hamiltonian. The projective case is described throughout the paper to illustrate the relative simplicity of the procedure as compared with the one in [M2].

Several results of independent interest are proved along the study. For example, in the appendix we prove that differential invariants of curves in flat $G / H$ with $G$ semisimple have order greater than 1 . In section 6 , Theorem 8 , we also describe the precise relation between classical and group theoretical moving frames. This relation was previously known only for some affine and projective geometries and was recently described by the author in the conformal case ([M3]). Work by Ochiai ([Oc]), although perhaps due to Cartan, shows that a moving frame $\rho\left(u^{(k)}\right) \in G$ can be locally factored as $\rho=\rho_{-1} \rho_{0} \rho_{1}$ with $\rho_{0}\left(u^{(k)}\right) \in G_{0}$ identified with an element of $\operatorname{GL}(n, \mathbb{R})$, where $n$ is the dimension of the manifold $G / H$. We show that we can identify $\rho_{0}$ with a matrix in $\operatorname{GL}(n, \mathbb{R})$ containing in columns a classical moving frame (a curve in the tangent manifold) along $u$. A similar situation can be found for some nonsemisimple cases in [M4].

As we said before, this is not a paper on the subject of completely integrable systems. We aim to define what we call geometric Poisson brackets and to directly relate geometric flows with Hamiltonian PDEs. Some new results have been already obtained using the brackets defined here. In [M6] the author found integrable systems associated to geometric flows of Lagrangian planes in symplectic $\mathbb{R}^{2 n}$. Still, the general classification of these geometric completely integrable systems is pending and includes the classification of compatible and nondegenerate geometric Poisson brackets. Although there are available tools to tackle given particular cases, the general study is still open. Integrable PDEs is also not the only application of the results presented here. In our last section, we describe on-going research and some open problems. The author would like to thank Professors Georgia Benkart, Peter Olver and Joel Robbin for continuous support and discussions.

2. Definitions and basic results. In this section we will give the basic definitions and results on flat homogeneous spaces associated to the action of semisimple Lie groups and their Cartan geometries. The definitions and results in this section are mainly stated as in $[\mathrm{Oc}]$ and $[\mathrm{Sh}]$.

Let $G$ be a Lie group and let $H$ be a closed subgroup. The homogeneous space $G / H$ is called flat of order 2 if the following two conditions hold true:

(a) the Lie algebra $\mathfrak{g}$ of $G$ has a graded Lie algebra structure

$$
\mathfrak{g}=\mathfrak{g}_{-1} \oplus \mathfrak{g}_{0} \oplus \mathfrak{g}_{1}
$$

(b) $\mathfrak{h}=\mathfrak{g}_{0} \oplus \mathfrak{g}_{1}$ is the Lie subalgebra corresponding to $H$.

The homogeneous space $G / H$ is called semisimple flat if $G$ is furthermore semisimple. It is well-known that a flat semisimple homogeneous space is flat of order $2([\mathrm{KO}])$.

The following results on the geometry of flat semisimple homogeneous spaces can be found in $[\mathrm{Oc}]$.

Proposition 1. Denote by $B$ the Killing form of $\mathfrak{g}$. Then

(1) $\mathfrak{g}_{-1} \oplus \mathfrak{g}_{1}$ and $\mathfrak{g}_{0}$ are perpendicular to each other with respect to $B$, so that both $B \mid \mathfrak{g}_{-1} \oplus \mathfrak{g}_{1}$ and $B \mid \mathfrak{g}_{0}$ are nondegenerate;

(2) $\mathfrak{g}_{-1}$ is the dual vector space of $\mathfrak{g}_{1}$ under the pairing $(x, z) \rightarrow B(x, z)$.

(3) There exists a unique element $e$ in $\mathfrak{g}_{0}$ such that

$$
[e, x]=-x, \quad \text { for } \quad x \in \mathfrak{g}_{-1},
$$




$$
\begin{aligned}
& {[e, y]=0, \quad \text { for } \quad y \in \mathfrak{g}_{0}} \\
& {[e, z]=z, \quad \text { for } \quad z \in \mathfrak{g}_{1} .}
\end{aligned}
$$

Now we introduce the group in the picture. Let $G_{1}$ (resp. $G_{-1}$ ) be the connected Lie subgroup of $G$ corresponding to $\mathfrak{g}_{1}$ (resp. $\mathfrak{g}_{-1}$ ). We define $G_{0}$ to be the normalizer of $\mathfrak{g}_{0}$ in $H$, that is, $G_{0}=\left\{a \in H \mid \operatorname{Ad}(a)\left(\mathfrak{g}_{0}\right)=\mathfrak{g}_{0}\right\}$.

Proposition 2. The exponential mapping exp $: \mathfrak{g}_{1} \rightarrow G_{1}$ and $\exp : \mathfrak{g}_{-1} \rightarrow G_{-1}$ are bijective. Furthermore, $G_{0}$ is also the normalizer of $\mathfrak{g}_{-1}$ in $H$ and $H$ is the semidirect product of $G_{0}$ and $G_{1}$.

The subgroup $G_{0}$ is called the linear isotropy subgroup of the homogeneous space $G / H$ and it is clearly locally bijective, via the exponential map, to $\mathfrak{g}_{0}$.

Example 1 . Consider the group $G=\operatorname{PSL}(n+1, \mathbb{R})$ acting on $\mathbb{R}^{n}$ projectively. That is, if $x \in \mathbb{R}^{n}$ we lift it to $\mathbb{R}^{n+1}$ as $(x, 1)$, we apply $g \in G$ and we project it back into $\mathbb{R}^{n}$. The isotropy subgroup of the origin is given by

$$
H=\left\{\left(\begin{array}{ll}
g_{0} & 0 \\
w & \alpha
\end{array}\right)\right\} \subset G
$$

where $\alpha \neq 0, \alpha \in \mathbb{R}$.

It is known that $\mathbb{R P}^{n} \simeq G / H$ is a flat semisimple homogeneous space. The Lie algebra $\mathfrak{g}$ splits as in (2.1) where

$$
\left(\begin{array}{cc}
0 & v \\
0 & 0
\end{array}\right) \in \mathfrak{g}_{-1},\left(\begin{array}{cc}
A & 0 \\
0 & a
\end{array}\right) \in \mathfrak{g}_{0},\left(\begin{array}{cc}
0 & 0 \\
w & 0
\end{array}\right) \in \mathfrak{g}_{1}
$$

and where

$$
e=\left(\begin{array}{ll}
0 & 0 \\
0 & 1
\end{array}\right)
$$

The corresponding subgroups are given by elements of the form

$$
\left(\begin{array}{ll}
I & * \\
0 & 1
\end{array}\right) \in G_{-1},\left(\begin{array}{cc}
\Theta & 0 \\
0 & \ell
\end{array}\right) \in G_{0},\left(\begin{array}{cc}
I & 0 \\
* & 1
\end{array}\right) \in G_{1}
$$

with $\ell \neq 0$ and $\operatorname{det} \Theta=\ell^{-1}$.

Definition 1. [Sh] A Cartan Geometry $(P, \omega)$ on a manifold $M$ modeled on $(G, H)$ consists of the following data:

(1) a smooth manifold $M$;

(2) a principal $\mathrm{H}$-bundle $P$ over $M$;

(3) a $\mathfrak{g}$-value 1-form on $P$ satisfying the following conditions

(i) for each point $p \in P$, the linear map $\omega_{p}: T_{p} P \rightarrow \mathfrak{g}$ is an isomorphism;

(ii) $\left(R_{h}\right)^{*} \omega=A d\left(h^{-1}\right) \omega$ for all $h \in H$;

(iii) $\omega(0, X)=X$ for all $X \in \mathfrak{h}$

where, as usual, $R_{h}$ denotes the right multiplication map, $h \in H$, Ad represents the Adjoint action of the group, and $(0, X)$ is a trivialization of the element in $P$ associated to $X \in \mathfrak{h}$. The form $\omega$ is usually called the Cartan Connection . 
If $(M, P, \omega)$ is a Cartan geometry, the $\mathfrak{g}$-valued 2 -form on $P$ given by

$$
\Omega=d \omega+\frac{1}{2}[\omega, \omega]
$$

is called the curvature. Equation (2.2) is called the structural equation.

If $\Omega=0$ we say the Cartan connection is flat. It is known (see [Oc]) that any flat Cartan connection on the principal bundle $G \rightarrow G / H$ is locally isomorphic to the connection defined by the Maurer-Cartan form of $G$. In this paper we will consider a semisimple flat homonegeous space with its standard Cartan connection.

Next we will describe the action of $G_{0}$ and $G_{1}$ on the bundle of frames. This description will connect us to the moving frame method described in the next section.

Definition 2. Let $M$ be a manifold. We fix a point in the manifold as the origin $o$. Let $V$ and $V^{\prime}$ be two neighborhoods of $o$ and let $U$ and $U^{\prime}$ be two neighborhoods of a point $x \in M$. Two diffeomorphisms $f: V \rightarrow U$ and $f^{\prime}: V^{\prime} \rightarrow U^{\prime}$ are said to define the same $r$-frame whenever $f^{\prime}(o)=f(o)=x$ and $f$ and $f^{\prime}$ have the same partial derivatives at $o$ up to order $r$. The $r$-frame given by $f$ is usually denoted by $j_{o}^{r}(f)$.

Let $\mathcal{F}^{r}(M)$ be the set of $r$-frames (or frames of $r^{\text {th }}$ order contact.) The set $\mathcal{F}^{r}(M)$ is a principal bundle over $M$ with projection $\pi\left(j_{o}^{r}(f)\right)=f(0)$. If $\operatorname{dim} M=n$, its structure group is denoted by $G^{r}(n)$ and it is given by the set of $r$-frames at $o \in M$ leaving $o$ invariant and acting by composition on the right (see $[\mathrm{K}]$ for more details.) The operation of the group is also composition. The bundle $\mathcal{F}^{1}(M)$ is the frame bundle and clearly $G^{1}(n)$ is isomorphic to $\operatorname{GL}(n, \mathbb{R})$. Finally two further points: first $\mathrm{GL}(n, \mathbb{R})$ can be considered as a subgroup of $G^{r}(n)$ if we consider the jets of linear maps. Also, there exists a natural projection of 2 -frames on 1 -frames and we will denote it by $\nu$

$$
\nu: \mathcal{F}^{2}(M) \rightarrow \mathcal{F}^{1}(M)
$$

In the context of flat semisimple homogenous spaces, each element $g \in G$ can be identified with the diffeomorphism of $M, \phi_{g}$, given by the action (or left multiplication) of $G$ on $M$. Thus, every $g \in G$ determines a jet $j_{o}^{r}\left(\phi_{g}\right) \in G^{r}(n)$. With this identification the following results are true and their proof can be found in [Oc].

THEOREM 1.

(1) $H=G_{0} \cdot G_{1}$ is a subgroup of $G^{2}(n)$;

(2) If we identify $G^{1}(n)$ with $\mathrm{GL}(n, \mathbb{R})$ then, as subgroups of $G^{2}(n), \nu(H)=G_{0}$, where $\nu$ is as in (2.3);

(3) $G \rightarrow G / H$ is an flat $H$-subbundle of $\mathcal{F}^{2}(G / H) \rightarrow G / H$.

(4) $\nu(G) \rightarrow G / H$ is a flat $G_{0}$-subbundle of $\mathcal{F}^{1}(G / H) \rightarrow G / H$.

Given $g \in G$, since the map $\mathfrak{g}_{-1} \oplus \mathfrak{g}_{0} \oplus \mathfrak{g}_{1} \rightarrow G$ taking $v=v_{-1}+v_{0}+v_{1}$ to $\exp \left(v_{-1}\right) \exp \left(v_{0}\right) \exp \left(v_{1}\right)$ is a local diffeomorphism, we can, locally, define a factorization

$$
g=g_{-1} g_{0} g_{1}
$$

such that $g_{i} \in G_{i}$. From (2) above we conclude that, if

$$
\phi_{g_{0} g_{1}}: G / H \rightarrow G / H
$$


takes $x$ to $g_{0} g_{1} x$, then $d \phi_{g_{1}}(o)=I$ and we can identify $d \phi_{g_{0}}(o) \in \operatorname{GL}(n, \mathbb{R})$ with $g_{0}$ itself, identifying this way $G_{0}$ with a subgroup of $\operatorname{GL}(n, \mathbb{R})$. The action of $G_{0}$ on the tangent to $G / H$ at $e$ (that is, $\mathfrak{g}_{-1}$ ) is given by the standard action of $\operatorname{GL}(n, \mathbb{R})$ on $\mathbb{R}^{n}$.

The following proposition can be found in [Oc] and will be used later in the paper.

Proposition 3. The subgroup $H=G_{0} \cdot G_{1}$ (as an element of $G^{2}(n)$ ) leaves $\mathfrak{g}_{-1} \oplus \mathfrak{g}_{0}$ (as contained in $\mathfrak{g}_{-1} \oplus \operatorname{gl}\left(\mathfrak{g}_{-1}\right)$ ) invariant. Its action onto $\mathfrak{g}_{-1} \oplus \mathfrak{g}_{0}$ is identical to the action of $H$ on $\mathfrak{g} / \mathfrak{g}_{1}$ induced by the Adjoint action $\operatorname{Ad}(a): \mathfrak{g} \rightarrow \mathfrak{g}, a \in H$.

The action in Proposition 3 is the action on $\mathfrak{g}_{-1}$ as $T_{o}(G / H)$ and on $g_{0}$ as $T_{I}\left(G^{1}(n)\right)$, where $I$ is the identity, induced by the action of $H$ on $G / H$ and $G^{1}(n)$ (this last one by composition), respectively. In particular, if $g_{0} \in G_{0}$, then the action on $T_{o}(G / H)$ induced by $\phi_{g_{0}}$ is given by the adjoint action of $G_{0}$ on $g_{-1}$. We will come back to this point in the next section.

3. Moving frames. In this section we will describe the method of moving frames as redesigned by Fels and Olver in [FO1] and [FO2].

Definition 3. Let $G$ be a Lie group acting on a manifold $M$. We say that $G$ acts (locally) effectively on subsets if, for every open subset $U \subset M$, the global isotropic subgroup of $U$ given by

$$
\{g \in G \text { such that } g \cdot s=s, \text { for all } s \in U\}
$$

is trivial, that is, equal to $\{e\}$. We say the group acts freely on $M$ if the isotropy subgroups $G_{x}=\{g \in G$ such that $g \cdot x=x\}$ are all trivial.

DeFINITION 4. Let $G$ be an $r$-dimensional Lie group acting on an $n$-dimensional manifold. We say that the group acts regularly on $M$ if all its orbits have the same dimension and each point $x \in M$ has arbitrarily small neighborhoods whose intersection with each orbit is a connected subset thereof. If the second condition in the definition is ommited we say the group acts semi-regularly.

If $G$ acts semi-regularly on $M$ a (local) cross-section is a $(n-s)$-dimensional submanifold $C \subset M$ such that $C$ intersects each orbit transversally. The implicit function theorem guarantees the existence of a transverse section through each point in $M$.

Definition 5. Given a transformation group $G$ acting on a manifold $M$, a moving frame is a smooth $G$-equivariant map

$$
\rho: M \rightarrow G
$$

Since we can consider either the right or the left action of $G$ on itself, we can talk about right or left moving frames. We are going to work with left moving frames. The following theorem determines which group actions admit a moving frame. Its proof can be found in [FO1].

THEOREM 2. If $G$ acts on $M$, then a moving frame exists in a neighborhood of a point $x \in M$ if, and only if $G$ acts freely and regularly near $x$. 
Perhaps the most interesting part of Fels and Olver's method is that it describes a simple way of constructing moving frames based on the so-called normalization equations.

Definition 6. Let $C \subset M$ be a cross-section to the $G$ orbits. The normalization equations associated with $C$ are the system of equations

$$
w=g \cdot z=c
$$

where $c \in C$.

It is quite simple to see that, if $G$ acts freely and $C$ is a regular cross section, then there is a unique solution $g=\rho(z)$ to the normalization equations, determining the right moving frame associated with $C$.

Moving frames are used to find a complete set of invariants of the action. These invariants can be found in two different ways.

THEOREM 3. If $\rho(x)$ is a moving frame, then the components of the map I : $M \rightarrow M$ defined by $I(x)=\rho(x) \cdot x$ provide a complete set of invariants for the group.

Therefore, the normalization procedure provides a simple direct method for determining invariants of free group actions. Unlike Lie's method, it does not require integrating any differential equation.

Next, we will describe the situation when the manifold $M$ is the jet space associated to curves in a homogeneous space. A variation of what will be defined below needs to be adopted whenever the group $G$ acts also on the independent variable, which is not the case at hand.

Definition 7. Given a manifold $M$, we define the $n$th order jet bundle $\mathcal{J}^{n}(M)$ to be the set of equivalence classes of curves under the equivalence relation of $n$th order contact. The fibers of $\pi^{n}: \mathcal{J}^{n} \rightarrow M$ are generalized Grassman manifolds (see $[\mathrm{O}]$.

We introduce local coordinates $u=\left(u^{\alpha}\right)$ on $M$. The induced coordinates in the jet bundle $\mathcal{J}^{n}(M)$ are denoted by $\left(x, u^{(n)}\right)$, where $x$ is the independent variable and where the components of $u^{(n)}$ are $u_{k}^{\alpha}$ representing the $k$ th derivative of the dependent variable $u^{\alpha}$ with respect to $x$, for any $k \leq n$. Any transformation group $G$ acting on $M$ preserves the order of contact between curves. Therefore, there is an induced action of $G$ on the $n$th order jet bundle $\mathcal{J}^{n}$ known as the $n$th prolongation of $G$. In our special case the prolonged action is locally given by

$$
g \cdot\left(x, u, u_{1}, \ldots, u_{n}\right)=\left(x, g \cdot u,(g \cdot u)_{1}, \ldots,(g \cdot u)_{n}\right)
$$

where, again, the subindex indicates the number of derivatives with respect to $x$. The invariants of the prolonged action are naturally called differential invariants. The best known differential invariants are the curvature and torsion for curves in Euclidean space.

Definition 8. A (nth order) differential invariant is a (locally defined) scalar function $I: \mathcal{J}^{n} \rightarrow \mathbb{R}$ which is invariant under the prolonged action of $G$.

The key element of Fels and Olver's method is that, for a high enough order of contact, a moving frame always exists for the prolonged action. In fact, a theorem by Ovsiannikov [Ov] (corrected by Olver in [O2]) states that if a group acts (locally) effectively on subsets, then there exists an integer $k_{0}$ such that the prolonged action of 
the given group is locally free on an open and dense subset of the $k_{0}$ th order jet space. This subset is formed by the so-called regular jets (see [FO1]). Direct application of Theorem 2 implies that, if the group acts (locally) effectively on subsets, then for $n$ large enough, there exists a moving frame defined on a neigbourhood of regular jets (that is, for generic curves). Moreover, this moving frame can be found solving the normalization equations (3.2) for some choice of transversal section.

Fels and Olver's method provide also a complete description of generating differential invariants. Let $\rho\left(u^{(k)}\right)$ be a moving frame. From Theorem 3 a complete set of differential invariants is contained in $\rho\left(u^{(k)}\right) \cdot u_{r}, r=0,1,2, \ldots$ But given the normalization equations, some of these differential invariants are constant. We call the constant invariants phantom differential invariants. The following theorem can be found in [FO2] (I have adapted the notation to our particular case).

THEOREM 4. A generating system of differential invariants consist of

(a) All non-phantom zeroth order invariants $\rho \cdot u$ and $x$.

(b) All non-phantom differential invariants $\left(\rho\left(u^{(k)}\right) \cdot u_{r}\right)^{\alpha}$ for which $\left(\rho\left(u^{(k)}\right)\right.$. $\left.u_{r-1}\right)^{\alpha}$ was phantom. (The expression ()$^{\alpha}$ represents the entry $\alpha$ as an element of $\mathbb{R}^{n}$.)

From this theorem, if $\rho\left(u^{(k)}\right) \cdot u_{r}=c_{r}$ has maximal rank, then all $r$ th order differential invariants will be phantom invariants and differential invariants will have higher order (or lower if some appeared first). This is the idea behind the method of used in the appendix to show that, the case at hand, there are no first order differential invariants and $\rho\left(u^{(k)}\right) \cdot u_{1}=c_{1}$ has maximal rank for some $c_{1} \neq 0$. It is also the same idea behind the method of Cartan polygons found in [G].

A second method to compute the invariants is to find the Serret-Frenet equations or moving coframe associated to a moving frame.

Definition 9. Suppose that $G$ acts freely and regularly on $\mathcal{J}^{k}$. Let $\rho: \mathcal{J}^{k} \rightarrow G$ be a (left) moving frame. We let $\zeta=\rho^{*} \omega$ denote the pull-back of the (left-invariant) Maurer-Cartan forms to $M$. Let $K d x=\omega\left(\rho_{x}\right) d x$ be its horizontal component. We call $K$ the Serret-Frenet equations or moving coframe associated with the given moving frame along the curve $u$.

The entries of the Serret-Frenet equations associated to a moving frame also provides a complete set of invariants of the group (see [FO1]), although there could be algebraic relations among its entries.

Definition 10. A vector $R: \mathcal{J}^{n} \rightarrow \mathbb{R}^{n}$ is said to be a relative differential invariant corresponding to the Jacobian multiplier if

$$
R\left(g \cdot u^{(k)}\right)=J_{g} R\left(u^{(k)}\right)
$$

where $J_{g}(x) \in \mathrm{GL}(n, \mathbb{R})$ is the Jacobian matrix of the map $\phi_{g}: M \rightarrow M, \phi_{g}(x)=g \cdot x$.

Along a curve, a nondegenerate matrix of relative differential invariants with Jacobian multiplier and a classical moving frame (a curve in the tangent bundle) are the same concept. The first one emphasizes the algebraic properties under the prolonged action of the group and the second one the geometric meaning.

4. Poisson Geometry of the spaces of Loops in the dual of a Lie algebra. Poisson reduction. This section is the last piece of our puzzle. In section 5 we will start putting sections 2,3 and 4 together. 
Let $G$ be a semisimple Lie group, and $\mathfrak{g}$ its Lie algebra. Let $\mathcal{L} G=C^{\infty}\left(S^{1}, G\right)$ be the group of Loops on $G$ and let $\mathcal{L} \mathfrak{g}=C^{\infty}\left(S^{1}, \mathfrak{g}\right)$ be its Lie algebra. Let $\mathcal{L}^{*}=$ $C^{\infty}\left(S^{1}, \mathfrak{g}^{*}\right)$ be its dual (it is not really its dual but what is called the regular part of the dual, dense in the dual of the algebra of Loops.) The space of Loops could be replaced by functions from $\mathbb{R}$ to $G$ vanishing at infinity, or any condition that ensures that no boundary terms will appear when we integrate by parts.

There exists a well known Poisson bracket defined on this space. If we have a functional $\mathcal{H}: \mathcal{L}^{*} \rightarrow \mathbb{R}$, its variational derivative, $\frac{\delta \mathcal{H}}{\delta M}(M)$, is given by an element of $\mathcal{L} \mathfrak{g}$ and it is defined by the Frechet derivative of the functional, namely

$$
\left.\frac{d}{d \epsilon}\right|_{\epsilon=0} \mathcal{H}(M+\epsilon V)=\int_{S^{1}} B\left(\frac{\delta \mathcal{H}}{\delta M}(M), V\right) d x
$$

where $B$ is the nondegenerate pairing between $\mathfrak{g}$ and $\mathfrak{g}^{*}$. (Notice that semi-simplicity of the algebra is a fundamental condition on the definition of this bracket.) If $\mathcal{H}$ and $\mathcal{G}$ are two functionals defined on $\mathcal{L}_{\mathfrak{g}}$, then their Poisson bracket is given by the formula

$$
\{\mathcal{H}, \mathcal{G}\}(M)=\int_{S^{1}} B\left(\frac{\delta \mathcal{G}}{\delta M}(M),\left(\frac{\delta \mathcal{H}}{\delta M}(M)\right)_{x}+a d^{*}(M)\left(\frac{\delta \mathcal{H}}{\delta M}(M)\right)\right) d x .
$$

To learn about the relationship between this Poisson bracket and the central extensions of $\mathcal{L}^{*}$ please see [PS]. In fact, the above is the Lie-Poisson bracket on the regular part of the dual of the Kac-Moody algebra of $G$ on $S^{1}$.

For us, perhaps the fundamental property of this Poisson bracket is the fact that its symplectic leaves (the leaves where the flow of Hamiltonian systems lie) coincide with the orbits in $\mathcal{L}_{\mathfrak{g}}$ under the action of the group of Loops $\mathcal{L} G$ given by

$$
A(g)(M)=g^{-1} g_{x}+A d^{*}(g) M
$$

whose infinitesimal action of the Lie algebra $\mathcal{L} \mathfrak{g}$ is given by

$$
a(N)(M)=N_{x}+a d^{*}(N)(M) .
$$

See [PS] for more information. If we view $\mathfrak{g}$ as a subalgebra of the algebra of matrices $\operatorname{gl}(m, \mathbb{R})$ and $G$ as a subgroup of $\mathrm{GL}(m, \mathbb{R})$, action (4.2) coincide with the action by conjugation of the group on operators of the form

$$
L=\frac{d}{d x}+M
$$

This action can also be viewed as the action on solutions of $\frac{d X}{d x}=X M$. Indeed, if $Y=X g$ with $g \in \mathcal{L} G$, then the operator (4.4) associated to $Y$ is given by

$$
\frac{d}{d x}+A(g)(M)
$$

Notice that, if $M$ is periodic, a solution $X$ will have a monodromy, that is, there exists $\Gamma \in G$ such that $X(x+T)=\Gamma X(x)$ for all $x$, where $T$ is the period. In fact, symplectic leaves of this bracket are known to be classified by the $G$-conjugation class of the monodromy.

We will now describe the Poisson reduction that will be implemented in the next section. The general reduction procedure that we will apply is identical to the one 
described in [MR]: Let $(\mathcal{P},\{\}$,$) be a Poisson manifold, Let \mathcal{M} \subset \mathcal{P}$ be a submanifold and $i: \mathcal{M} \rightarrow \mathcal{P}$ the inclusion. Let $\left.E \subset T \mathcal{P}\right|_{\mathcal{M}}$ be a subbundle of the tangent to $\mathcal{P}$ restricted to $\mathcal{M}$. Assume the following conditions are satisfied:

(a) $E \cap T \mathcal{M}$ is integrable and define a foliaton on $\mathcal{M}$. We will call the foliation $\Phi$.

(b) The space of leaves is a manifold and $\pi: \mathcal{M} \rightarrow \mathcal{M} / \Phi$ is a submersion.

(c) $E$ leaves $\{$,$\} invariant. That is, if \mathcal{H}, \mathcal{G}: \mathcal{P} \rightarrow \mathbb{R}$ are smooth functions on $\mathcal{P}$ with differentials vanishing on $E$, then the differential of $\{\mathcal{H}, \mathcal{G}\}$ also vanish on $E$. One says that $E$ preserves the Poisson bracket $\{$,$\} .$

Definition 11. We say $(\mathcal{P}, \mathcal{M}, E,\{\}$,$) is Poisson reducible if \mathcal{M} / \Phi$ has a Poisson structure, $\{,\}_{R}$ such that for any smooth $h, g: \mathcal{M} / \Phi \rightarrow \mathbb{R}$ and for any smooth extensions $\mathcal{H}, \mathcal{G}$ of $f \circ \pi$ and $g \circ \pi$ with differentials vanishing on $E$ we have

$$
\{\mathcal{H}, \mathcal{G}\} \circ i=\{h, g\}_{R} \circ \pi .
$$

The following theorem can be found in $[\mathrm{MR}]$ for the finite dimensional case, although it can be applied to infinite dimensional cases, as the authors state. In either case, the geometric picture is very clear.

Theorem 5. (Reduction Theorem) Assume $(\mathcal{P}, \mathcal{M}, E,\{\}$,$) satisfy assumptions$ (a)-(b)-(c) and let $P: T^{*} \mathcal{P} \rightarrow T \mathcal{P}$ be the map associating to each variational derivative its Hamiltonian vector field. Then, $(\mathcal{P}, \mathcal{M}, E,\{\}$,$) is Poisson reducible if, and$ only if

$$
P\left(E^{0}\right) \subset T \mathcal{M}+E
$$

5. Geometric Poisson brackets on the space of differential invariants of curves in a flat semisimple homogeneous space. This section contains one of the key results of this paper. We will show that, if $G / H$ is a flat semisimple homogeneous manifold, then the space of Serret-Frenet equations can be identified with a quotient of $L \mathfrak{g}^{*}$. This fact enables us to readily reduce the Poisson bracket defined in the previous section to the manifold of invariant coframes (or differential invariants of curves in $G / H)$. From now on we will assume that $G / H$ is a flat semisimple homogeneous space.

The first step in the identification of the quotient is to prove a lemma introducing a constant element $\Lambda \in \mathfrak{g}_{-1}$. The rest of the choices will depend on $\Lambda$ and its isotropy subgroup.

Lemma 1. Given any moving frame $\rho$ along a curve $u: I \rightarrow G / H$, where $I$ is some open interval in $\mathbb{R}$, denote by $K=\omega\left(\rho_{x}\right)$ its associated Serret-Frenet equation ( $\omega$ is the Cartan connection). Let $K=K_{-1}+K_{0}+K_{1}$ be the decomposition of $K$ associated to (2.1).

There exists a left moving frame $\rho$ such that $K_{-1}=\Lambda$ is constant, independent from $x$. Furthermore, if $\rho=\rho_{-1} \rho_{0} \rho_{1}$ is the local factorization of the frame as in (2.4), then $\rho_{-1}$ can be identified with $u$ itself.

Proof. The proof of this lemma is based on the normalization procedure used in the Fels-Olver method. Following that method, for $m$ high enough, we can choose a 
constant transversal section $C$ to the orbits of the prolonged action of $G$ on $\mathcal{J}^{m}(G / H)$, and we can obtain a moving frame by simply solving the normalization equation

$$
g \cdot u^{(m)}=c
$$

$c \in C$, for $g$. Assume $g=g_{1} g_{0} g_{-1}$ is the local factorization of $g$ and assume $c=$ $\left(c_{0}, c_{1}, c_{2}, \ldots, c_{m}\right)$ with the normalization equations given by $(g \cdot u)_{k}^{\alpha}=c_{k}^{\alpha}, c_{k}^{\alpha}$ constant. We choose $c_{0}=o=\left[x_{0}\right] \in G / H$. The equation

$$
g_{1} g_{0} g_{-1} \cdot u=o
$$

has maximal rank and can be solved the following way: we first solve for $g_{-1} \cdot u=o$ which can be uniquely solved for $g_{-1}$ in terms of $u$ because of the transitivity of the action. In fact, the usual (local) identification of $M$ with $G_{-1}$ (or with a transverse section of $G / H)$ is given by uniquely solving $u=g_{u} \cdot o$ for $g_{u} \in G_{-1}$. Under this identification $g_{-1}^{-1}=g_{u}$ will identify with $u$. We call $g_{-1}=\hat{\rho}_{-1}$. Since $g_{1} g_{0} \in H$ leaves $c_{0}=o$ invariant, this first step determines the $G_{-1}$ component of the moving frame.

Next, we look at the first jet space. We choose $c_{1}$ to be a constant element in the tangent of our homogeneous space. With the previous choice of $\hat{\rho}_{-1}=g_{-1}=g_{u}^{-1}$ we solve for $\rho_{0}$ so that we satisfy condition

$$
\left.\left(g_{1} g_{0} g_{-1} \cdot u\right)_{1}\right|_{g_{-1}=g_{u}^{-1}}=c_{1} .
$$

The above is equal to

$$
d \phi_{g_{1}}(o) d \phi_{g_{0}}(o) d \phi_{g_{-1}}(u) u_{1}=c_{1} .
$$

As we explained after Theorem $1, \phi_{g_{1}}, g_{1} \in G_{1}$, does not affect $\mathcal{F}^{1}(M)$ and so $d \phi_{g_{1}}(o)=I$. Now, notice that if we consider $u \in V_{u} \subset G / H$ locally identified with $g_{u} \in W_{u} \subset G_{-1}$, then $g_{-1} \cdot u$ is simply left multiplication $g_{-1} g_{u}$, and also $\hat{\rho}_{-1}=g_{u}^{-1}$. Therefore $d \phi_{g_{-1}}(u) u_{1}$ is simply the left invariant identification of $u_{1} \in T_{g \cdot u} G_{-1}$ with an element in the algebra $T_{e} G_{-1}=\mathfrak{g}_{-1}$. We denote this identification as $g_{u_{1}}$, and so $\left.d \phi_{g_{-1}}(u) u_{1}\right|_{g_{-1}=g_{u}^{-1}}=g_{u_{1}} \in \mathfrak{g}_{-1}$.

With this in mind, we only need to solve for

$$
d \phi_{g_{0}}(o) g_{u_{1}}=c_{1} \text {. }
$$

Here we apply the appendix's result stating that there are no first order differential invariants of curves in flat semisimple homogenous spaces. From the appendix, the rank of the equation above is maximal for a generic curve and for some choice of constant $c_{1}$. Hence, we can partially solve for $g_{0}$ to satisfy the equation. Since the equation is linear, we have $c_{1} \neq 0$. One would go on to higher orders of differentiation to complete the moving frame. How high one needs to go will depend on the manifold.

The result of this normalization process is a right invariant moving frame. Thus, the left invariant moving frame will be its inverse $\rho=\hat{\rho}^{-1}=\hat{\rho}_{-1}^{-1} \hat{\rho}_{0}^{-1} \hat{\rho}_{1}^{-1}=\rho_{-1} \rho_{0} \rho_{1}$. Notice that $\rho_{-1}=g_{u}$ is identified with $u$.

Finally it is not hard to see that, since $\omega$ is the left invariant Maurer-Cartan form, the moving coframe $K$ can be split into

$$
K=A d\left(\rho_{1}^{-1} \rho_{0}^{-1}\right)\left(\omega\left(\left(\rho_{-1}\right)_{x}\right)\right)+A d\left(\rho_{1}^{-1}\right)\left(\omega\left(\left(\rho_{0}\right)_{x}\right)\right)+\omega\left(\left(\rho_{1}\right)_{x}\right)
$$

so that the $\mathfrak{g}_{-1}$ component is the $\mathfrak{g}_{-1}$ component of $\operatorname{Ad}\left(\rho_{1}^{-1}\right)\left[\operatorname{Ad}\left(\rho_{0}^{-1}\right)\left({ }^{*} \omega\left(\left(\rho_{-1}\right)_{x}\right)\right)\right]$. A very basic use of Campbell's formula shows that the $\mathfrak{g}_{-1}$ component is

$$
K_{-1}=A d\left(\rho_{0}^{-1}\right)\left(\omega\left(\left(\rho_{-1}\right)_{x}\right)\right) .
$$


On the other hand, $\omega\left(\left(\rho_{-1}\right)_{x}\right)$ is the left-invariant identification of $u_{1} \in \mathcal{J}^{1}$ with an element of $\mathfrak{g}_{-1}$ (that is, $\left.\omega\left(\left(\rho_{-1}\right)_{x}\right)=g_{u_{1}}\right)$. Application of Proposition 3 allows us to conclude that $K_{-1}$ is constant since $\operatorname{Ad}\left(\rho_{0}^{-1}\right)\left(\omega\left(\left(\rho_{-1}\right)_{x}\right)\right)=\operatorname{Ad}\left(\hat{\rho}_{0}\right)\left(\omega\left(\left(\rho_{-1}\right)_{x}\right)\right)$ is given by the action of $\hat{\rho}_{0}$ on $u_{-1}$, which, by Proposition 3 equals

$$
\operatorname{Ad}\left(\hat{\rho}_{0}\right)\left(\omega\left(\left(\rho_{-1}\right)_{x}\right)\right)=\left.d \phi_{g_{0}}(0)\right|_{g_{0}=\hat{\rho}_{0}} g_{u_{1}}=c_{1} .
$$

We call

$$
K_{-1}=\Lambda=c_{1}
$$

and we end the proof of the lemma.

Incidentally, one can find other relations between $K$ and the constants $c_{r}$ 's. For example, we can check with a short straightforward calculation similar to the one above that $\left[K_{0}, K_{-1}\right]=c_{2}$.

Comment: From the discussion in the proof of the lemma, $K_{-1}$ contains the first order differential invariants of our curve. From our appendix, flat homogeneous semisimple manifolds do not have invariants of that order and therefore $K_{-1}$ is constant (all first order differential invariants are phantom differential invariants). There exists known nonsemisimple flat cases for which Poisson brackets of the kind presented here exist ([M1], [M4]). In these cases we do have one first order differential invariants, namely the arc-length. But in the reduction process applied to these cases one needs to ask for the arc-length to be constant and to be preserved by the geometric evolutions under consideration. This condition has two consequences: first we don't have non-phantom differential invariants of first order any longer, second we transform our bracket into a bracket not on $\mathfrak{g}^{*} \oplus \mathbb{R}$ but rather on $\mathfrak{g}^{*}$, effectively gaining semisimplicity. After introducing the change, a modification of the reduction presented in this section also works in Euclidean, special affine and other cases, see [M1] and [M4].

EXAMPLE 2. We go back to the projective case. If $g \in \operatorname{PSL}(n+1, \mathbb{R})$ is given by

$$
\left(\begin{array}{cc}
I & 0 \\
w & 1
\end{array}\right)\left(\begin{array}{cc}
A & 0 \\
0 & a
\end{array}\right)\left(\begin{array}{ll}
I & v \\
0 & 1
\end{array}\right)=\left(\begin{array}{cc}
A & A v \\
w A & a+w A v
\end{array}\right)
$$

with $a=\operatorname{det}(A)^{-1}$, one can describe the action of $\operatorname{PSL}(n+1, \mathbb{R})$ on $u \in \mathbb{R} P^{n}$ as

$$
g \cdot u=\frac{A(u+v)}{w A(u+v)+a} .
$$

Following Fels and Olver's method we normalize the zero order action, namely we set

$$
g \cdot u=0
$$

and we solve for $v$, namely $v=-u$. This choice determines

$$
\hat{\rho}_{-1}(u)=\left(\begin{array}{cc}
I & -u \\
0 & 1
\end{array}\right)
$$

The standard local identification of $\mathbb{R} P^{n}$ with $\operatorname{PSL}(n+1, \mathbb{R}) / H$ is given by

$$
u \rightarrow g_{u}=\left(\begin{array}{cc}
I & u \\
0 & 1
\end{array}\right)=\hat{\rho}_{-1}^{-1}(u)=\rho_{-1}(u)
$$


With this choice of $\hat{\rho}_{-1}$, after we evaluate the normalizations, we have that the denominator of the action is given by $w A(u+v)+a=a$. We then proceed with the normalizations that will partially determine $A$ and $a$. Indeed, the action on the first jet is given by

$$
\frac{A u_{1}}{w A(u+v)+a}-\frac{A(u+v)\left(w A u_{1}\right)}{(w A(u+v)+a)^{2}} .
$$

If we use the normalization (5.1) and set the above equals $e_{n}$, we obtain the relation

$$
a A^{-1} e_{n}=u_{1}
$$

for some choice of $\hat{\rho}_{0}=\rho_{0}^{-1}=\left(\begin{array}{cc}A & 0 \\ 0 & a\end{array}\right)$. The identification of $G_{0}$ with $\operatorname{GL}(n, \mathbb{R})$ is given by identifying $\left(\begin{array}{cc}A & 0 \\ 0 & a\end{array}\right)$ with $a^{-1} A$. This information suffices to find the $K_{-1}$ component of the moving coframe, which equals

$$
K_{-1}=A d\left(\left(\begin{array}{cc}
A & 0 \\
0 & a
\end{array}\right)\right)\left(\begin{array}{cc}
I & -u \\
0 & 1
\end{array}\right)\left(\begin{array}{cc}
0 & u_{1} \\
0 & 0
\end{array}\right)=\left(\begin{array}{cc}
0 & a^{-1} A u_{1} \\
0 & 0
\end{array}\right)=\left(\begin{array}{cc}
0 & e_{n} \\
0 & 0
\end{array}\right)=\Lambda
$$

For simplicity, we will identify $\mathcal{L} \mathfrak{g}^{*}$ with $\mathcal{L} \mathfrak{g}$ using the standard nondegenerate pairing defined by integrating the Killing form (so that $A d^{*}(g)$ becomes $A d\left(g^{-1}\right)$, for example). Let $\mathcal{M}$ be the submanifold of $\mathcal{L}^{*}$ given by

$$
\mathcal{M}=\mathcal{L}\left(\mathfrak{g}_{0} \oplus \mathfrak{g}_{1}\right) \oplus S_{\Lambda}
$$

where $S_{\Lambda}$ is the subspace of $\mathcal{L}_{-1}$ given by

$$
S_{\Lambda}=\left\{\beta \Lambda, \text { for any } \beta \text { positive } \beta \in C^{\infty}\left(S^{1}\right)\right\} .
$$

Next, consider $\mathcal{L} L_{0} \subset \mathcal{L} G_{0}$ to be the subgroup of $\mathcal{L} G_{0}$ preserving $S_{\Lambda}$ (its isotropy subgroup). Let $L$ be the semidirect product of $\mathcal{L} L_{0}$ and $\mathcal{L} G_{1}$,

$$
L=\mathcal{L} L_{0} \cdot \mathcal{L} G_{1} \subset \mathcal{L} H .
$$

Let $\mathfrak{l}$ be the Lie algebra associated to $L$. The subbundle of $\left.T \mathcal{L}_{\mathfrak{g}}{ }^{*}\right|_{\mathcal{M}}$ that will be used in the reduction process will be the one generated by the orbits of $L$ using the action given by (4.2). Indeed, let

$$
\left.E \subset T \mathcal{L}_{\mathfrak{g}}\right|_{\mathcal{M}}
$$

be the distribution generated by the Hamiltonian vector fields corresponding to functionals $\mathcal{F}$ such that $\frac{\delta \mathcal{F}}{\delta M}(M) \in \mathfrak{l}$ for any $M \in \mathcal{M}$. It is known that $E$ is integrable and its leaves coincide with the action of $L$ on $\mathcal{M}$ as in (4.2).

These are our players. We will first check that a transverse section to an open set of the leaves of $E$ is given by the submanifold of differential invariants along curves in $G / H$ (which will be defined shortly). We will then readily check that the Lie-Poisson bracket defined in section 4 can be Poisson reduced to $\mathcal{M} / E$.

Lemma 2. Consider the action on the right of $G$ on itself. Then, the induced action of $\mathcal{L} G$ on $\omega\left(\rho_{x}\right)$, where $\rho$ is a moving frame, is as in (4.2). That is, if $\rho$ is a moving frame and $g \in \mathcal{L} G$, then

$$
\omega\left((\rho g)_{x}\right)=A(g) K=\omega\left(g_{x}\right)+A d\left(g^{-1}\right) K
$$


where $K$ is the moving coframe associated to $\rho$ and $\omega$ is the left-invariant Cartan connection.

Proof. The proof is straightforward from properties of the Cartan connection.

Comment: If $m \in \mathcal{M}$, locally we can find a map $\rho: J \rightarrow G$ for some interval $J$ containing 0 and such that $\omega\left(\rho_{x}\right)=m$ where $\omega$ is the Maurer-Cartan form in the group and $\rho(0)=e \in G$. To produce $\rho$ we need to extend $m(x)$ to a (x-dependent) left invariant vector field in the group, say $X_{m}(\rho, x)$. We then locally solve the equations

$$
\frac{d \rho}{d x}=X_{m}(\rho(x), x)
$$

If we choose a faithful representation of $\mathfrak{g}$ and we take $G$ to be a Lie group associated to $\mathfrak{g}$ and a subgroup of $\mathrm{GL}(n, \mathbb{R})$, then $\omega\left(\rho_{x}\right)=\rho^{-1} \rho_{x}$ and $\rho$ would be the fundamental matrix solution of the system $\frac{d \rho}{d x}=\rho(x) m(x)$. Furthermore, if $m(x)$ is periodic in $x, \rho$ will always have a monodromy, that is, there will be $\Gamma \in G$ such that $\rho(x+T)=\Gamma \rho(x)$ for any $x$.

TheOREM 6. Let $L \subset \mathcal{L} G$ be the subgroup given as in (5.6). Given $m \in \mathcal{M}$, let $\rho$ be such that $\omega\left(\rho_{x}\right)=m$ with $\rho(0)=e$. Let $\rho=\rho_{-1} \rho_{0} \rho_{1}$ be the local factorization described in previous sections. Then, there exists a unique $g \in L$ such that $\rho g=\rho_{K}$, where $\rho_{K}$ is the moving frame associated to the curve $u$ defined by $\rho_{-1}$ under the identification described in the proof of Lemma 1. Furthermore, there exists and open set $U \subset \mathcal{M}$ such that the quotient $U / E$ can be locally identified with $\mathcal{K}$, the space of moving coframes.

Proof. Let $\rho: J \rightarrow G$ be such that $\omega\left(\rho_{x}\right) \in \mathcal{M}$, and let $\rho=\rho_{-1} \rho_{0} \rho_{1}$ be its local unique factorization (guaranteed locally around $e$ ). Let $g_{0} \cdot g_{1} \in L$, where $g_{0} \in \mathcal{L} L_{0}$, $g_{1} \in \mathcal{L} G_{1}$. Then

$$
\rho g=\rho_{-1}\left[\rho_{0} g_{0}\right]\left[g_{0}^{-1} \rho_{1} g_{0} g_{1}\right] .
$$

Since $G_{0}$ is the Stabilizer of $\mathfrak{g}_{1}$, we have that $g_{0}^{-1} \rho_{1} g_{0} g_{1} \in G_{1}, \rho_{0} g_{0} \in G_{0}$ and $\rho_{-1} \in$ $G_{-1}$.

Let $u: J \rightarrow G / H$ be the curve represented by $\rho_{-1}$, that is $\rho_{-1}=g_{u}$. We will show that $g$ as above can be chosen so that $\rho g=\rho_{K}$, were $\rho_{K}$ is the moving frame associated to $u$ as in Lemma 1. Indeed, we need to choose $g_{0}$ so that $\rho_{0} g_{0}=\left(\rho_{0}\right)_{K}$ and $g_{1}$ so that $g_{0}^{-1} \rho_{1} g_{0} g_{1}=\left(\rho_{1}\right)_{K}$. These two relations uniquely determine $g_{0}$ and $g_{1}$. After this determination one needs to prove:

(i) $g_{0}=\rho_{0}^{-1}\left(\rho_{0}\right)_{K}$ leaves $S_{\Lambda}$ invariant and hence it is in $L_{0}$, and

(ii) $g=g_{0} g_{1}$ is periodic, that is $\rho$ and $\rho_{K}$ have the same monodromy.

Indeed, if $\rho$ and $\tilde{\rho}$ are such that $\omega\left(\rho_{x}\right)=m \in \mathcal{M}$ and $\omega\left(\tilde{\rho}_{x}\right)=\tilde{m} \in \mathcal{M}$, and such that $\rho_{-1}=\tilde{\rho}_{-1}$, then one has

$$
\operatorname{Ad}\left(\rho_{0}^{-1}\right)\left(\omega\left(\left(\rho_{-1}\right)_{x}\right)\right)=\beta \Lambda
$$

for some $\beta>0$, since $\operatorname{Ad}\left(\rho_{0}^{-1}\right)\left(\omega\left(\left(\rho_{-1}\right)_{x}\right)\right)$ is the $\mathfrak{g}_{-\tilde{\sigma}_{\tilde{\beta}}}$ component of $\omega\left(\rho_{x}\right)$ as we saw in Lemma 1. Likewise for $\tilde{\rho}_{0}$ with a different choice $\tilde{\beta}$. Hence

$$
A d\left(\tilde{\rho}_{0}^{-1}\right) \operatorname{Ad}\left(\rho_{0}\right)(\Lambda)=\frac{1}{\beta} \operatorname{Ad}\left(\tilde{\rho}_{0}^{-1}\right) \omega\left(\left(\rho_{-1}\right)_{x}\right)=\frac{\tilde{\beta}}{\beta} \Lambda,
$$


which shows that $g_{0}$ leaves $S_{\Lambda}$ invariant. Furthermore, if $\omega\left(\rho_{x}\right)=m \in \mathcal{M}$, then $m$ is periodic and there exists $\Gamma \in G$ such that $\rho(x+T)=\Gamma \rho(x)$ for all $x$. If we factor $\rho$ out we have

$$
\Gamma \rho_{-1} \rho_{0} \rho_{1}=\Gamma g_{u} \rho_{0} \rho_{1}=g_{\Gamma \cdot u} \hat{\rho}_{0} \hat{\rho}_{1}=\rho(x+T)=g_{u}(x+T) \rho_{0}(x+T) \rho_{1}(x+T)
$$

using Corollary 1 (notice that the proof of the corollary does not depend on results here). Therefore $g_{\Gamma \cdot u}=g_{u}(x+T)$ and, since $g_{u}(x+T)=g_{u_{T}}$ with $u_{T}(x)=u(x+T)$, we also have $u(x+T)=\Gamma \cdot u(x)$. From the definition of prolonged action $\Gamma \cdot u^{(k)}(x)=$ $u^{(k)}(x+T)$ also. The normalization equations gives us

$$
u^{(k)}(x)=\rho_{K}(x) \cdot c_{k}
$$

and so

$$
\Gamma \cdot u^{(k)}(x)=\Gamma \rho_{K}(x) \cdot c_{k}=u^{(k)}(x+T)=\rho_{K}(x+T) \cdot c_{k},
$$

for any $k$. From here we can conclude that $\rho_{K}(x+T)=\Gamma \rho_{K}(x)$ and so $K=\omega\left(\left(\rho_{K}\right)_{x}\right)$ will be periodic since $\rho_{K}$ and $\rho$ have the same monodromy.

To end the proof we need to show that $\mathcal{K}$ can be identified locally with $U / E$, where $U$ is an open subset of $\mathcal{M}$. This is an immediate consequence of the continuous dependence of solutions of ODEs on their equations, which guarantees that, if an orbit in $\mathcal{M}$ intersects $\mathcal{K}$, a nearby orbit will remain in $\mathcal{M}$ (that is $\beta>0$ ) and will intersect $\mathcal{K}$.

EXAMPLE 3. In the projective case, the moving frame is well known. Its original description is due to Wilczynski $[\mathrm{W}]$ and it is as follows: given a curve in $\mathbb{R}^{n}$ assume that the curve is nondegenerate, that is, that the Wronskian of the functions $u_{1}, u_{2}, \ldots, u_{n}$ is positive. Let's denote this Wronskian by $W$. We next lift $u$ to $\mathbb{R}^{n+1}$ uniquely so that the Wronskian of the lift is constant and equals one. Namely $v=(\phi u, \phi)^{T}$ with $\phi=W^{-\frac{1}{n+1}}$. Let $\rho$ be the matrix having $v_{n}, v_{n-1}, \ldots, v_{1}, v$ in columns. From the construction $\rho \in \operatorname{PSL}(n+1)$. The map associating to each curve $u$ the matrix $\rho$ is the classical projective (left) moving frame for $u$. Its associated coframe is easily seen to be of the form

$$
\omega\left(\hat{\rho}_{x}\right)=\hat{\rho}^{-1} \hat{\rho}_{x}=\left(\begin{array}{ccccc}
0 & 1 & 0 & \ldots & 0 \\
\hat{\mathbf{k}}_{n-1} & 0 & 1 & \ldots & 0 \\
\vdots & & \ddots & & \vdots \\
\hat{\mathbf{k}}_{1} & 0 & \ldots & 0 & 1 \\
\hat{\mathbf{k}}_{0} & 0 & \ldots & 0 & 0
\end{array}\right)
$$

and $\hat{\mathbf{k}}_{i}, i=0,1, \ldots, n-1$ are the well-known Wilczynski invariants, independent differential invariants that generate any other projective differential invariant for $u$.

A change of frame replacing parts of the $\rho_{0}$ factor and the $\rho_{1}$ factor can be found to transform this Serret-Frenet equations into the more convenient one

$$
\omega\left(\rho_{x}\right)=\rho^{-1} \rho_{x}=\left(\begin{array}{ccccc}
0 & 1 & 0 & \ldots & 0 \\
0 & 0 & 1 & \ldots & 0 \\
\vdots & & \ddots & & \vdots \\
0 & 0 & \ldots & 0 & 1 \\
\mathbf{k}_{0} & \mathbf{k}_{1} & \ldots & \mathbf{k}_{n-1} & 0
\end{array}\right)
$$


One can see that this moving frame can be obtained using Fels and Olver's method as above. The interested reader can fill in the details of this change, we will avoid further explanations.

For the projective case we choose

$$
\mathcal{M}=\left\{\left(\begin{array}{cc}
* & \beta e_{n} \\
* & *
\end{array}\right), \beta>0, \beta \in C^{\infty}\left(S^{1}\right)\right\}
$$

and

$L=\left\{\left(\begin{array}{cc}A_{0} & 0 \\ 0 & a_{0}\end{array}\right)\left(\begin{array}{cc}I & 0 \\ * & 1\end{array}\right), a_{0}^{-1}=\operatorname{det} A_{0}, \quad A_{0}=\left(\begin{array}{cc}A_{1} & 0 \\ * & a_{1}\end{array}\right), a_{1}>0\right\} \subset \mathcal{L P S L}(n, \mathbb{R})$.

The theorem above shows that any matrix in $\mathcal{M}$ can be taken to one of the form (5.9) using the action (4.2), with $g \in L$.

Next theorem will finally show that the Poisson bracket described in the previous section can be Poisson reduced using $\mathcal{M}$ and $E$. The resulting bracket will be a Hamiltonian structure defined on the manifold of Serret-Frenet equations (or differential invariants) associated to curves in the homogeneous manifold $G / H$.

Theorem 7. Let $\mathcal{M}$ and $E$ be as in (5.4) and (5.7). Let $\{$,$\} be the Poisson$ bracket (4.1) defined on $\mathcal{L}_{\mathfrak{g}}{ }^{*}$. Then $\left(\mathcal{L}^{*}, \mathcal{M}, E,\{\},\right)$ is Poisson reducible and there exists a Poisson bracket $\{,\}_{R}$ defined on the manifold of coframes $\mathcal{K}$. The reduced bracket is defined by relation (4.5).

Proof. First of all, we have seen already that conditions (a) and (b) needed to apply the Reduction Theorem are satisfied since the map

$$
\pi: \mathcal{M} \rightarrow \mathcal{K}
$$

is given by $m \rightarrow A(g) m$ where $g$ is as in Theorem 6. Next, (c) is also satisfied. Indeed, if $\frac{\delta F}{\delta M}(M)$ and $\frac{\delta G}{\delta M}(M)$ belong to $E^{0}$ clearly their Hamiltonian vector fields $P\left(\frac{\delta F}{\delta M}(M)\right)$ and $P\left(\frac{\delta G}{\delta M}(M)\right)$ belong to $\mathfrak{l}^{0}$ along $\mathcal{M}$. Therefore, if $R$ is such that $\frac{\delta R}{\delta M}(M) \in \mathfrak{l}$ we have

$$
\{F, R\}(M)=\{G, R\}(M)=0
$$

for any $M \in \mathcal{M}$, and so

$$
\frac{\delta\{G, R\}}{\delta M}(M), \frac{\delta\{F, R\}}{\delta M}(M) \in(T \mathcal{M})^{0} .
$$

If $\mathfrak{l}_{0}$ is the Lie algebra associated to the isotropy subgroup $L_{0}$ and $\mathfrak{l}_{0}^{0}$ represents its annihilator within $\mathfrak{g}_{0}$, we have $\mathfrak{l}^{0}=\mathfrak{g}_{1} \oplus \mathfrak{l}_{0}^{0}$ and so $\mathcal{L} \mathfrak{l}^{0} \subset T_{M} \mathcal{M}$ as vector subspaces. From here $\left(T_{M} \mathcal{M}\right)^{0} \subset \mathcal{L l}$ and, using Jacobi's identity

$$
\{\{G, F\}, R\}(M)=0
$$

for any $M \in \mathcal{M}$. That is, (c) is satisfied.

Finally, condition (4.6) is also satisfied since $E^{0}$ is generated by elements $F$ such that $P(F)(M) \in \mathfrak{l}^{0}$. Therefore

$$
P\left(E^{0}\right) \subset \mathcal{L l}^{0} \subset T_{M} \mathcal{M}
$$


for any $M \in \mathcal{M}$.

If one wishes to obtain the reduced bracket explicitly, the reduction process might not seem to be very practical. In many specific examples though, obtaining an explicit formula is not too complicated, as the projective example shows. The conformal and Euclidean cases were worked out in [M1] and [M3].

EXAMPLE 4. Let $\mathcal{K} \subset \mathcal{M}$ be the submanifold of matrices as in (5.9). In the projective case, the reduced Poisson bracket can be found as follows: if $\mathcal{H}$ is any extension of $h: \mathcal{K} \rightarrow \mathbb{R}$ constant of the leaves of $E$, then

$$
H=\frac{\delta \mathcal{H}}{\delta M}=\left(\begin{array}{cc}
H_{0} & \mathbf{h} \\
H_{1} & h_{0}
\end{array}\right)
$$

where $\mathbf{h}=\left(\frac{\delta h}{\delta \mathbf{k}_{i}}\right)$ is the variational derivative of $h$. Since $\mathcal{H}$ must be constant on the leaves of $E$ we have that

$$
(H)_{x}+[K, H]=\left(\begin{array}{cc}
\left(H_{0}\right)_{x}+\left[\alpha, H_{0}\right]+e_{n} H_{1}-\mathbf{h k}^{T} & \mathbf{h}_{x}+\alpha \mathbf{h}+h_{0} e_{n}-H_{0} e_{n} \\
\left(H_{1}\right)_{x}+\mathbf{k}^{T} H_{0}-H_{1} \alpha-h_{0} \mathbf{k}^{T} & \left(h_{0}\right)_{x}+\mathbf{k}^{T} \mathbf{h}-H_{1} e_{n}
\end{array}\right)
$$

should belong to $\mathfrak{g}_{1} \oplus \mathfrak{l}_{0}^{0}$, where $K$ is as in (5.9), that is $K$ equals

$$
K=\left(\begin{array}{cc}
\alpha & e_{n} \\
\mathbf{k}^{T} & 0
\end{array}\right), \quad \alpha=\left(\begin{array}{ccccc}
0 & 1 & 0 & \ldots & 0 \\
0 & 0 & 1 & \ldots & 0 \\
\vdots & \vdots & \ddots & \ddots & \vdots \\
0 & 0 & \ldots & 0 & 1 \\
0 & 0 & 0 & 0 & 0
\end{array}\right)
$$

Hence, we obtain the following equations for $H_{0}, H_{1}$ and $h_{0}$

$$
\begin{aligned}
H_{1} e_{n} & =\left(h_{0}\right)_{x}+\mathbf{k}^{T} \mathbf{h} \\
\left(H_{0}-h_{0} I\right) e_{n} & =\mathbf{h}_{x}+\alpha \mathbf{h} \\
\left(H_{0}\right)_{x}+\left[\alpha, H_{0}\right]+e_{n} H_{1}-\mathbf{h k}^{T} & =\left(\begin{array}{cccc}
0 & 0 & \ldots & 0 \\
\vdots & \vdots & \vdots & \vdots \\
0 & 0 & \ldots & 0 \\
* & \ldots & * & *
\end{array}\right)
\end{aligned}
$$

It is not hard to see that these equations determine $H_{0}-h_{0} I$ (notice that $h_{0}=-t r H_{0}$ ) and $H_{1} e_{n}$ in terms of $\mathbf{k}$ and $\mathbf{h}$ through algebraic manipulation (that is, no integration needed). Since we know the bracket will be independent of the extension from $\mathcal{M}$ to $\mathcal{L} \mathfrak{g}^{*}$, other entries in $H_{1}$ will not appear in the final expression. Indeed, once we solve for $H_{0}$ and $H_{1} e_{n}$ using the equations above, the reduced bracket is given by

$$
\begin{aligned}
\{h, g\}_{R}(K)=\int_{S^{1}} & \left.\left(H_{1}\right)_{x}-H_{1} \alpha+\mathbf{k}^{T}\left(H_{0}-h_{0} I\right)\right) \mathbf{g} \\
& +\left(e_{n}^{T}\left(\left(H_{0}\right)_{x}+\left[\alpha, H_{0}\right]+e_{n} H_{1}-\mathbf{h k}^{T}\right)\right) G_{0} e_{n} d x .
\end{aligned}
$$

But, from the equations above we know that $G_{0} e_{n}=\mathbf{g}_{x}+\alpha \mathbf{g}+g_{0} e_{n}$. Notice that the last entry of $e_{n}^{T}\left(\left(H_{0}\right)_{x}+\left[\alpha, H_{0}\right]+e_{n} H_{1}-\mathbf{h k}^{T}\right)$ vanishes since the trace of $H_{x}+[K, H]$ 
is zero. Hence, we can ignore $g_{0} e_{n}$. The reduced Hamiltonian vector field associated to $h$ is then given by

$$
\begin{gathered}
\xi_{h}^{R}=\mathbf{k}^{T}\left(H_{0}-h_{0} I\right) \\
-\frac{d}{d x}\left(e_{n}^{T}\left(\left(H_{0}\right)_{x}+\left[\alpha, H_{0}\right]\right)-h_{n} \mathbf{k}^{T}\right)+\left(e_{n}^{T}\left(\left(H_{0}\right)_{x}+\left[\alpha, H_{0}\right]\right)-h_{n} \mathbf{k}^{T}\right) \alpha,
\end{gathered}
$$

where $h_{n}$ is the $n$-entry of $\mathbf{h}$.

The fact that the bracket (4.1) with $\mathfrak{g}=\operatorname{sl}(n+1, \mathbb{R})$ was Poisson reducible to the space $\mathcal{K}$ of periodic matrices of the form (5.9) was originally proved algebraically by Drinfeld and Sokolov in [DS] and later linked to the geometry of curves in $\mathbb{R P}^{n}$ in [M2]. Although the original procedure used in [DS] and [M2] seems quite different than the one presented here (and the one in $[\mathrm{DS}]$ is purely algebraic), one can easily show that, in the $\operatorname{sl}(n+1, \mathbb{R})$ case, they are indeed equivalent. The manifold $\mathcal{M}$ and foliation $E$ used in Drinfel'd and Sokolov's paper are different from the ones presented here but it is not hard to see that the resulting reduced bracket is the same. In those papers the manifold used for reduction was

$$
\mathcal{M}_{1}=\Gamma+\mathcal{B}
$$

where

$$
\Gamma=\left(\begin{array}{ccccc}
0 & 1 & 0 & \ldots & 0 \\
0 & 0 & 1 & \ldots & 0 \\
\vdots & \vdots & \ddots & \ddots & \vdots \\
0 & 0 & \ldots & 0 & 1 \\
0 & 0 & \ldots & \ldots & 0
\end{array}\right)
$$

where $\mathcal{B} \subset \mathcal{L} \operatorname{sl}(n+1, \mathbb{R})$ is given by lower triangular matrices. The foliation involved in the reduction is described by $L_{1}$ the same way ours is described by $L$, where $L_{1}$ equals the identity plus strictly lower triangular periodic matrices. Clearly $\mathcal{M}_{1} \subset \mathcal{M}$ and $E_{1} \subset E$. It was also proved that $\mathcal{M}_{1} / E_{1}$ is locally equal to our $\mathcal{M} / E$. Since we are reducing in both cases the same Poisson bracket on the dual of the Kac-Moody algebra of $\operatorname{PSL}(n+1, \mathbb{R})$, it is simple to conclude that the reduced Poisson brackets coincide in both cases.

Drinfel'd and Sokolov showed that the reduced Poisson bracket coincides with the so-called Adler-Gel'fand-Dikii bracket or second Hamiltonian structure for generalized $\mathrm{KdV}$ equations and so is ours in the $\operatorname{PSL}(n+1, \mathbb{R})$ case. Their brackets in cases other than $\operatorname{PSL}(n+1, \mathbb{R})$ are not in general equal to the geometric brackets obtained here. For example, in the $O(n+1,1)$ case the resulting quotient in [DS] has dimension less than the number of independent differential invariants needed to generate differential invariants of curves in the Möbius sphere (the flat conformal geometry case). Therefore, their reduced bracket cannot coincide with ours. It would be interesting to study the precise relation between both brackets and the consequences for completely integrable systems of KdV type.

6. Hamiltonian systems induced by geometric evolutions. In this section we want to answer the following question: Let $u(t, x)$ be a family of curves in the homogeneous space $G / H$. Assume $u(t, x)$ are solutions of the evolution

$$
u_{t}=Z\left(u, u_{1}, u_{2}, \ldots\right)
$$


where $u_{i}=\frac{d^{i} u}{d x^{i}}$. Assume further than evolution (6.1) is invariant under the action of $G$, that is, assume $G$ takes solutions to solutions. In that case there exists a naturally induced evolution on the space of differential invariants of the curves. This evolution will give us a natural evolution on the manifold $\mathcal{K}$ of coframes along $u$.

$$
\mathbf{k}_{t}=Y\left(\mathbf{k}, \mathbf{k}_{1}, \mathbf{k}_{2}, \ldots\right)
$$

where $\mathbf{k}_{i}=\frac{d^{i} \mathbf{k}}{d x^{i}}$. We want to determine which invariant evolutions of the form (6.1) will produce evolutions of the form (6.2) which are Hamiltonian with respect to the reduced bracket presented in the previous section. How simple this relation is will depend on the choice of frame, as we explain below.

First of all, a standard result in invariant theory (see for example [O]) states that equation (6.1) is invariant under the action of the group if and only if

$$
u_{t}=R \mathbf{r}
$$

where $R$ is a nondegenerate matrix of relative differential invariants with Jacobian multiplier (or a matrix formed by a classical moving frame) and where $\mathbf{r}$ is a vector whose entries are differential invariants. Our next results will bring this general invariant evolution description into the group picture.

First of all, the precise relation between moving frames and classical moving frames is not known in general. Fels and Olver described the known cases (Euclidean and projective) in section 7 of [FO1]. The author further described the relation in the conformal case in [M3]. The following theorem establishes the relation in the general flat semisimple case.

THEOREM 8. Under the identifications made in Lemma 1, if $\rho$ is a moving frame associated to a curve $u$, with $\rho=\rho_{-1} \rho_{0} \rho_{1}$ and $\rho_{-1}=g_{u}$, then $\rho_{0}$ as an element of $G L(n, \mathbb{R})$ is a nondegenerate matrix of relative differential invariants with Jacobian weight.

Proof. Indeed, let $\phi_{g}, g \in G$, be the map defined by left multiplication, $\phi_{g}$ : $V \rightarrow G$ where $V \subset G_{-1}$ is a neighbourhood of $e \in G_{-1}$ identified locally with a neighbourhood of $o \in M$. If we decompose $\phi_{g}(x)$ into its $G_{-1}, G_{0}$ and $G_{1}$ components

$$
\phi_{g}(x)=g x=g_{-1}(x) g_{0}(x) g_{1}(x)
$$

we know from (2) in Theorem 1 that $g_{0}(x)$ can be identified with the matrix associated to $d \phi_{g x}(o)=d \phi_{g}(x) d \phi_{x}(o)$. Having this in mind, let $\rho=\rho_{-1} \rho_{0} \rho_{1}$ be the moving frame from Lemma 1. Since $\rho$ is a left-invariant moving frame

$$
\rho\left(g \cdot u^{(k)}\right)=g \rho\left(u^{(k)}\right)
$$

where $g \cdot u^{(k)}$ is the prolonged action. On the other hand, as we discussed above, $g \rho_{-1}\left(u^{(k)}\right) \rho_{0}\left(u^{(k)}\right) \rho_{1}\left(u^{(k)}\right)=g_{-1}(u) g_{0}(u) g_{1}(u) \rho_{0} \rho_{1}$. Since $G_{0}$ is the Stabilizer of $G_{1}$, $g_{1}(u) \rho_{0}=\rho_{0} \hat{g}_{1}(u)$ for some $\hat{g}_{1} \in G_{1}$. Therefore

$$
g \rho_{-1} \rho_{0} \rho_{1}=g_{-1}(u) g_{0}(u) g_{1}(u) \rho_{0} \rho_{1}=g_{-1}(u) g_{0}(u) \rho_{0} \hat{g}_{1}(u) \rho_{1}
$$

and so

(6.4) $g_{-1}(u)=\rho_{-1}\left(g \cdot u^{(k)}\right), \quad g_{0}(u) \rho_{0}\left(u^{(k)}\right)=\rho_{0}\left(g \cdot u^{(k)}\right), \quad \hat{g}_{1}(u) \rho_{1}\left(u^{(k)}\right)=\rho_{1}\left(g \cdot u^{(k)}\right)$. 
Since $g_{0}$ is identified with the element of $\operatorname{GL}(n, \mathbb{R})$ defined by the matrix associated to $d \phi_{g}\left(\rho_{-1}\right) d \phi_{\rho_{-1}}(o)$, and since $d \phi_{\rho_{-1}}(o)=I$ as pointed out in Lemma 1 , we have that $g_{0}(u)$ is given by the Jacobian of $\phi_{g}$ at $u$. Hence $\rho_{0}$ can be identified with an invertible matrix of relative differential invariant with Jacobian weight.

The following corollary was used in the previous section and it is an immediate consequence of (6.4) above.

Corollary 1. Assume $\tilde{\rho}(x) \in G$ for all $x$ and assume we are close enough to $e \in$ $G$ so that there is a unique factorization $\tilde{\rho}=\tilde{\rho}_{-1} \tilde{\rho}_{0} \tilde{\rho}_{1}$. Assume that $\rho_{-1}=\tilde{\rho}_{-1}=g_{u}$ as in Lemma 1. Let $\Gamma \in G$ be also close to e, so that $\Gamma \tilde{\rho}=\hat{\rho}_{-1} \hat{\rho}_{0} \hat{\rho}_{1}$. Then $\hat{\rho}_{-1}=g_{\Gamma \cdot u}$.

Proof. Let $\rho_{-1}=g_{u}$ and let $\rho$ be the moving frame corresponding to $u$ and found using Fels ad Olver's method. Let $\rho=\rho_{-1} \rho_{0} \rho_{1}$ be its factorization. From (6.4) above $\Gamma \rho_{-1} \rho_{0} \rho_{1}=g_{\Gamma \cdot u} \hat{\rho}_{0} \hat{\rho}_{1}$ which implies $\Gamma \rho_{-1}=g_{\Gamma \cdot u} \nu$ for some $\nu(x) \in H$. Thus

$$
\Gamma \tilde{\rho}=g_{\Gamma \cdot u} \nu \tilde{\rho}_{0} \tilde{\rho}_{1}
$$

and since $\nu \tilde{\rho}_{0} \tilde{\rho}_{1}(x)=\hat{\rho}_{0} \hat{\rho}_{1} \in H$ we obtain the result.

Lemma 3. Under the identifications made in Lemma 1, if $\rho$ is the moving frame associated to a curve $u$ found in that lemma and $\rho=\rho_{-1} \rho_{0} \rho_{1}$ with $\rho_{-1}=g_{u}$, then an invariant equation of the form (6.3) can be written as

$$
\omega\left(\left(\rho_{-1}\right)_{t}\right)=A d\left(\rho_{0}\right) v_{r}
$$

where $v_{r} \in \mathfrak{g}_{-1}$ is the element in $\mathfrak{g}_{-1}$ defined by some invariant vector $\mathbf{r}$ if we left invariantly identify $\mathfrak{g}_{-1}$ with the tangent to $G / H$, and where $\omega\left(\left(\rho_{-1}\right)_{t}\right) d t$ is the $t$ horizontal component of the pull back of the the Cartan connection by $\rho_{-1}(t, x)$ (that is $\left.\rho_{-1}^{-1}\left(\rho_{-1}\right)_{t}\right)$.

Proof. This proof is very simple if we apply the previous theorem. The left invariant identification of $u_{t}$ with an element in $\mathfrak{g}_{-1}$ is given by $\omega\left(\left(\rho_{-1}\right)_{t}\right)$ since $\omega$ is defined by the Maurer-Cartan form of $G$. Also, $\rho_{0}$ as an element of $\operatorname{GL}(n, \mathbb{R})$ identifies with a nondegenerate matrix of relative invariants. The action of $G_{0}$ (as subgroup of $\mathrm{GL}(n, \mathbb{R}))$ on $\mathfrak{g}_{-1}$ is given by the adjoint action. Hence, writing an invariant evolution of the form (6.3) in the algebra $\mathfrak{g}_{-1}$ results in equation (6.5), if we choose as $R$ the matrix given by $\rho_{0}$.

ExAmple 5. In the $\operatorname{PSL}(n+1, \mathbb{R})$ projective case, the first moving frame is easily seen to be

$$
\rho\left(u^{(k)}\right)=\left(\begin{array}{cccc}
(u \phi)^{(n)} & \ldots & (u \phi)^{\prime} & u \phi \\
\phi^{(n)} & \ldots & \phi^{\prime} & \phi
\end{array}\right)=\left(\begin{array}{cc}
I & u \\
0 & 1
\end{array}\right)\left(\begin{array}{cc}
A_{1} & 0 \\
0 & \phi
\end{array}\right)\left(\begin{array}{cc}
I & 0 \\
v_{1} & 1
\end{array}\right)
$$

$\phi=W^{-\frac{1}{n+1}}$ as before and where $v=\phi^{-1}\left(\phi^{(n)} \ldots \phi^{\prime} \quad \phi\right)$ and $A=$

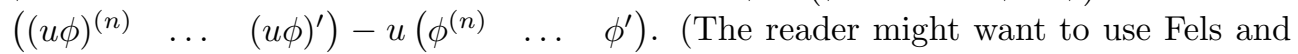
Olvers' method of normalization to quickly obtain this formula in the case $n=2$.) In the more convenient choice of second frame (5.9), we need to change $A_{1}$ and $v_{1}$ conveniently. In any case, the $G_{0}$ component $\left(\begin{array}{cc}A & 0 \\ 0 & \phi\end{array}\right)$ is identified with $\phi^{-1} A \in \mathrm{GL}(n, \mathbb{R})$. 
If we write (6.5) we obtain

$$
\left(\begin{array}{cc}
0 & -u \\
0 & 0
\end{array}\right)\left(\begin{array}{cc}
0 & u_{t} \\
0 & 0
\end{array}\right)=\left(\begin{array}{cc}
A & 0 \\
0 & \phi
\end{array}\right)\left(\begin{array}{cc}
I & \mathbf{r} \\
0 & 1
\end{array}\right)\left(\begin{array}{cc}
A^{-1} & 0 \\
0 & \phi^{-1}
\end{array}\right)
$$

that is

$$
\left(\begin{array}{cc}
0 & u_{t} \\
0 & 0
\end{array}\right)=\left(\begin{array}{cc}
0 & \phi^{-1} A \mathbf{r} \\
0 & 0
\end{array}\right)
$$

Evolution $u_{t}=\phi^{-1} A \mathbf{r}$, where $\mathbf{r}$ is a vector of differential invariants, that is depending on $\mathbf{k}$ and its derivatives, is the most general form of an invariant evolution under the projective action of $S L(n+1, \mathbb{R})$.

Our next step towards the classification of geometric evolutions is to write down a formula for the evolution induced on the manifold of moving coframes $\mathcal{K}$ by an invariant evolution of curves of the form (6.3). Once we write down the formula, its relation to the reduced bracket will become clear.

THEOREM 9. Let u be a two parameter family of curves solution of an invariant evolution of the form (6.3). Then, their associated moving coframes are solution of the equation

$$
K_{t}=N_{x}+[K, N]
$$

where $N=N_{1}+N_{0}+N_{-1} \in \mathfrak{g}$ is such that $N_{-1}=v_{r}$ is the left invariant identification of $\mathbf{r}$ with an element in $\mathfrak{g}_{-1}$.

Proof. As we said in Section 2, $G \rightarrow G / H$ is a $H$-principal bundle with a flat Cartan connection given by the Maurer-Cartan form on $G$. The structural equation is given by

$$
d \omega+\frac{1}{2}[\omega, \omega]=0
$$

Let $\rho$ be a moving frame along $u$ and let $\rho_{x}$ and $\rho_{t}$ represent the vector fields $\frac{d}{d x}$ and $\frac{d}{d t}$ along the two parameter family of curves $\rho$ in $G$. If we evaluate the structural equation on these two fields along $\rho$ we obtain

$$
\frac{d}{d t} \omega\left(\rho_{x}\right)-\frac{d}{d x} \omega\left(\rho_{t}\right)+\omega\left(\left[\rho_{x}, \rho_{t}\right]\right)=\left[\omega\left(\rho_{x}\right), \omega\left(\rho_{t}\right)\right] .
$$

Since $\omega$ is the Cartan connection, $\omega\left(\rho_{x}\right)=K$ is the associated moving coframe. Let's call $N=\omega\left(\rho_{t}\right)$ and notice that $t$ and $x$ are independent variables so, as fields on $G$, $\left[\rho_{x}, \rho_{t}\right]=0$. From here (6.7) becomes

$$
K_{t}=N_{x}+[K, N]
$$

which is (6.6). Assume $\rho=\rho_{-1} \rho_{0} \rho_{1}$. Then, as we saw in previous sections, if $N=$ $N_{-1}+N_{0}+N_{1}$

$$
N_{-1}=A d\left(\rho_{0}^{-1}\right) \omega\left(\left(\rho_{-1}\right)_{t}\right) .
$$

From (6.5) we have $N_{-1}=v_{r}$. 
Using the previous theorem and the definition of the reduced Poisson bracket we will be able to give a geometric interpretation of $\mathbf{r}$ in the Hamiltonian picture. We will show that, if $u$ induces a reduced Hamiltonian evolution on $K$ with Hamiltonian $h$, then $\mathbf{r}$ can be identified with the component tangent to $G / H$ of the variational derivative of an extension of the Hamiltonian constant on the leaves of the foliation used for the reduction. But first, as a point of reference, we will look into the situation in the projective case.

ExAmple 6. Assume $u_{t}=\phi^{-1} A \mathbf{r}$ is a projective geometric evolution and let $\mathcal{K}$ be the submanifold of $\mathcal{L} \operatorname{sl}(n+1, \mathbb{R})$ given by matrices of the form (5.9). If we denote by $\mathbf{k}=\left(\mathbf{k}_{1}, \ldots, \mathbf{k}_{n}\right)$,

$$
\alpha=\left(\begin{array}{cccc}
0 & 1 & \ldots & 0 \\
\vdots & \ddots & \ddots & \vdots \\
0 & \ldots & 0 & 1 \\
0 & \ldots & 0 & 0
\end{array}\right)
$$

and if we write

$$
N=\left(\begin{array}{cc}
N_{0} & \mathbf{r} \\
N_{1} & n_{0}
\end{array}\right)
$$

we can write $(6.8)$ as

$$
\left(\begin{array}{cc}
0 & 0 \\
\mathbf{k}_{t}^{T} & 0
\end{array}\right)=\left(\begin{array}{cc}
\left(N_{0}\right)_{x}+\left[\alpha, N_{0}\right]-\mathbf{r k}^{T}+e_{n} N_{1} & \mathbf{r}_{x}+\alpha \mathbf{r}-\left(N_{0}-n_{0} I\right) e_{n} \\
\left(N_{1}\right)_{x}-N_{1} \alpha+\mathbf{k}^{T}\left(N_{0}-n_{0} I\right) & \mathbf{k}^{T} \mathbf{r}+\left(n_{0}\right)_{x}-N_{1} e_{n}
\end{array}\right) .
$$

We can solve these equations for $N_{0}$ and $N_{1}$ in terms of $\mathbf{k}$ and $\mathbf{r}$ and their derivatives, and we can substitute the values we obtain in the entry for $\mathbf{k}_{t}$ to obtain the evolution of the curvatures. In fact, the above implies the following equations hold true

$$
\begin{aligned}
N_{1} e_{n} & =\left(n_{0}\right)_{x}-\mathbf{k}^{T} \mathbf{r} \\
\left(N_{0}-n_{0} I\right) e_{n} & =\mathbf{r}_{x}+\alpha \mathbf{r} \\
\left(N_{0}\right)_{x}+\left[\alpha, N_{0}\right]+e_{n} N_{1}+N_{1} e_{n} I-\mathbf{r} \mathbf{k}^{T}-\mathbf{k r} I & =0 .
\end{aligned}
$$

Compare these equations to equations (5.10). If $\mathbf{h}=\mathbf{r}$ then $N_{0}=H_{0}, n_{0}=h_{0}$ and so will the last entries of $H_{1}$ and $N_{1}$, since the equations are identical. Furthermore, the last row of the last equation above will also determine $N_{1}$ completely in terms of $\mathbf{k}$ and $\mathbf{r}$ so that

$$
N_{1}=e_{n}^{T}\left(-\left(N_{0}\right)_{x}-\left[\alpha, N_{0}\right]-\mathbf{r k}^{T}\right) .
$$

The evolution induced on the projective invariants is

$$
\mathbf{k}_{t}^{T}=\left(N_{1}\right)_{x}-N_{1} \alpha+\mathbf{k}^{T}\left(N_{0}-n_{0} I\right)
$$

which, with the value of $N_{1}$ above, coincide with the reduced Hamiltonian evolution given in (5.14). That is, the condition for the u-evolution to induce a Hamiltonian evolution on the differential invariants is that the invariant coefficients of the u-evolution, $\mathbf{r}$, coincide with the variational derivative of some Hamiltonian $h: \mathcal{K} \rightarrow \mathbb{R}$. In that case, the evolution of the invariants is Hamiltonian with Hamiltonian functional $h$. 
In general, we cannot expect to have such a simple relationship. The simplicity in the projective case relies on the fact that $T \mathcal{K}$ lies on $\mathfrak{g}_{1}$, the dual to $\mathfrak{g}_{-1}$, which is itself the tangent to $G / H$ at $e$. That is, if $h: \mathcal{K} \rightarrow \mathbb{R}$ is a Hamiltonian, any extension of $h$ to $\mathcal{L} \mathfrak{g}^{*}$ will have $\mathbf{h}$ in the $\mathfrak{g}_{-1}$ component of its variational derivative $H$, where $\mathbf{h}$ is the variational derivative of $h$. It is this tangential component that we must identify with $\mathbf{r}$ to make the $\mathbf{k}$ evolution Hamiltonian.

Definition 12. We say that a functional $h: \mathcal{K} \rightarrow \mathbb{R}$ is local if it depends on $\mathbf{k}$ and its derivatives. We say $\mathcal{K}$ is an affine subspace of $\mathcal{L} \mathfrak{g}$ if its entries are either constant or independent differential invariant generators.

In general we need to assume that local Hamiltonians exist. The non-local cases can be often dealt with formally (see [MS], [M1] and [M3] in the natural cases), but some care needs to be taken and we will avoid the complication here. All known examples have moving frames for which $\mathcal{K}$ is an affine subspace of $\mathcal{L} \mathfrak{g}$, but its general existence has not been proved yet.

THEOREM 10. Let $u(t, x)$ be a family of curves solution of an invariant evolution of the form (6.3), where $\mathbf{r}$ is a vector depending on $\mathbf{k}$ and its derivatives. Assume $\mathcal{K}$ is an affine subspace of $\mathcal{L} \mathfrak{g}$. Assume that there exists a local functional $h: \mathcal{K} \rightarrow \mathbb{R}$ and a local extension $\mathcal{H}: \mathcal{M} \rightarrow \mathbb{R}$ constant on the leaves of the foliation $\Phi$ used in our reduction. Assume further than $\frac{\delta \mathcal{H}}{\delta M}=H=H_{-1}+H_{0}+H_{1}$ with $H_{-1}=v_{r}$.

Then, the evolution induced by (6.3) on $\mathbf{k}$ is a Hamiltonian evolution with respect to the reduced bracket $\{,\}_{R}$, with associated Hamiltonian given by $h$.

Proof. Assume $h: \mathcal{K} \rightarrow \mathbb{R}$ and $g: \mathcal{K} \rightarrow \mathbb{R}$ are two local functionals with two local extensions $\mathcal{H}: \mathcal{M} \rightarrow \mathbb{R}, \mathcal{G}: \mathcal{M} \rightarrow \mathbb{R}$ constant on the leaves of $E$. If we denote by $H=\frac{\delta \mathcal{H}}{\delta M}(K)$ and $G=\frac{\delta \mathcal{G}}{\delta M}(K)$ we know that $H_{x}+[K, H], G_{x}+[K, G] \in \mathfrak{l}^{0}=\mathcal{L} \mathfrak{g}_{1} \oplus \mathcal{L} \mathfrak{l}_{0}^{0}$. Assume $\hat{\mathfrak{l}}_{0}$ is a complement to $\mathfrak{l}_{0}$, as vector subspaces, within $\mathfrak{g}_{0}$ so that $g_{0}=\mathfrak{l}_{0} \oplus \hat{\mathfrak{l}}_{0}$ and $\mathfrak{l}_{0}^{0}=\hat{\mathfrak{l}}_{0}^{*}$, the dual to $\hat{\mathfrak{l}}_{0}$. Let $H=H_{1}+H_{0}+H_{-1}$ be the decomposition given by the gradation and let us denote by $\hat{H}_{0}$ the component of $H_{0}$ in $\hat{\mathfrak{l}}_{0}$. Assume the same decomposition for $K$ and $G$. Since $H_{x}+[K, H] \in \mathfrak{l}^{0}$ we have

$$
\left(H_{-1}\right)_{x}+\left[K_{-1}, H_{0}\right]+\left[K_{0}, H_{-1}\right]=0
$$

which determines $\hat{H}_{0}$ in terms of $K_{-1}, K_{0}$ and $H_{-1}$. In fact, because $\mathcal{H}$ is local, $\hat{H}_{0}$ can be written in terms of derivatives of $H_{-1}$ and $\mathbf{k}$. That is, we can write

$$
\hat{H}_{0}=\mathcal{D}\left(H_{-1}\right)
$$

for some differential operator $\mathcal{D}$ with coefficients depending on $\mathbf{k}$ and its derivatives. Let us now write the reduced bracket. Since $H_{x}+[K, H] \in \mathfrak{l}^{0}$

$$
\begin{gathered}
\xi_{h}(g)=-\xi_{g}(h)=\{h, g\}_{R}(K)=\int_{S^{1}} B\left(H_{x}+[K, H], G\right) d x \\
=-\int_{S^{1}} B\left(\mathcal{D}^{*}\left(\left(G_{0}\right)_{x}+\left[K_{-1}, G_{1}\right]+\left[K_{1}, G_{-1}\right]+\left[K_{0}, G_{0}\right]\right)\right. \\
\left.+\left(G_{1}\right)_{x}+\left[K_{1}, G_{0}\right]+\left[K_{0}, G_{1}\right], H_{-1}\right) d x
\end{gathered}
$$

where $\mathcal{D}^{*}$ denotes the adjoint differential operator to $\mathcal{D}$ with respect to $B$.

Now we go back to the curve evolution $u_{t}=R \mathbf{r}$. We know that the induced evolution on the curvatures is given by $K_{t}=N_{x}+[K, N]$ with $N=\omega\left(\rho_{t}\right)$ and 
$N_{-1}=v_{r}$. Notice that, since $K_{-1}=\Lambda$ is constant

$$
\left(N_{-1}\right)_{x}+\left[K_{-1}, N_{0}\right]+\left[K_{0}, N_{-1}\right]=0
$$

and so $\hat{N}_{0}$ is determined as a function of $N_{-1}=v_{r}$, its derivatives, and the derivatives of $\mathbf{k}$. In fact

$$
\hat{N}_{0}=\mathcal{D}\left(N_{-1}\right)
$$

where $\mathcal{D}$ is given as in (6.10).

Since $K_{t} \in T \mathcal{K}$, for any local extension $\mathcal{G}$ of $g$, the variational derivative of $g$, denoted by $\mathbf{g}$, will appear in the $T \mathcal{K}^{*}$ components of $G$. That is, regardless of what the other components of $G$ are, we have that, considering $\mathbf{k}_{t}$ as a vector field on $\mathcal{K}$

$$
\mathbf{k}_{t}(g)=\int_{S^{1}} B\left(G, K_{t}\right) d x=\int_{S^{1}} B\left(G, N_{x}+[K, N]\right) d x=-\int_{S^{1}} B\left(G_{x}+[K, N], N\right) d x .
$$

Using the fact that $G_{x}+[K, G] \in \mathfrak{l}^{0}$, the above equals

$$
\begin{aligned}
-\int_{S^{1}} B\left(\left(G_{0}\right)_{x}+\left[K_{-1}, G_{1}\right]\right. & \left.+\left[K-1, G_{-1}\right]+\left[K_{0}, G_{0}\right], \hat{N}_{0}\right) \\
+ & B\left(\left(G_{1}\right)_{x}+\left[K_{1}, G_{0}\right]+\left[K_{0}, G_{1}\right], N_{-1}\right) d x .
\end{aligned}
$$

if we compare this expression to (6.11) using (6.12) we obtain that, if $v_{r}=N_{-1}=H_{-1}$ both vector fields $\xi_{h}$ and $\mathbf{k}_{t}$ are equal.

7. Compatible Hamiltonian structures. As we explained in the introduction, one of the main applications of infinite dimensional Poisson Geometry to completely integrable systems is the use of Poisson brackets to integrate the equations. Most completely integrable systems are biHamiltonian, that is, Hamiltonian with respect to two different and compatible Poisson structures. If one of these structures is symplectic, it can be formally inverted and a sequence of symmetries can be then found by recursion. This family of symmetries integrates the equation (see $[\mathrm{AF}]$ ). Because of this application we will briefly describe possible Poisson structures compatible with the geometric Poisson structure presented before.

In the projective case generalized $\mathrm{KdV}$ are biHamiltonian and one of the brackets (the so-called Second Hamiltonian KdV structure) is the reduced one presented here. It is then natural to ask whether or not the reduced Poisson bracket $\{,\}_{R}$ has one (or more) compatible Poisson structures. Indeed, the bracket (4.1) has a well-known compatible companion, namely the bracket

$$
\{\mathcal{H}, \mathcal{G}\}(L)=\int_{S^{1}} B\left(\frac{\delta \mathcal{G}}{\delta L}, a d^{*}\left(H_{1}\right)\left(\frac{\delta \mathcal{H}}{\delta L}\right)\right) d x
$$

where $H_{1} \in \mathfrak{g}^{*}$ is any constant element. Therefore, we need to look for constant elements $H_{1}$ such that (7.1) reduces to $\mathcal{K}$. Such a reduction would provide a compatible Poisson structure to $\{,\}_{R}$ and with it the possibility of finding integrable systems associated to flat geometries. (For the reader looking for a thrill, what about finding a completely integrable system associated to $E_{6}$ or $E_{7}$ ?) The study of possible reductions of (7.1) provided a known Hamiltonian structure for generalized KdV equation in the projective case, the so-called first KdV structure ([DS]) and also three compatible Hamiltonian structures in the $n=3$ Euclidean case ([MS]), including the ones 
integrating the Vortex filament flow and modified KdV. As far as the author knowns, except for the planar case, conformal and the general case are still largely open. Integrable systems in the planar case have been classified in [CQ1], [CQ2], although they have not been linked to these Hamiltonian structures yet. One of the main obstacles to the swift application of these results is the identification of possible reductions and non-degenerate brackets among the reduced ones. Another one is, of course, the fact that in many geometries moving frames and differential invariants are not known! These problems are already within reach of being solved in a case-by-case basis. It is still not clear how to classify geometric integrable systems in general.

A quick review of several examples show that there is not a clear general condition that guarantees the reduction of (7.1). One of the reduction conditions will restrict the possible values of $H_{1}$. Indeed, one needs

$$
\left\{E^{0}, \cdot\right\}(M) \subset T_{M} \mathcal{M}
$$

for any $M \in \mathcal{M}$. An element in $E^{0}$ can have $\mathfrak{g}_{-1}$ component and so, if we want to guarantee $a d^{*}\left(H_{1}\right)(H(M)) \in \mathfrak{g}_{1} \oplus \mathfrak{g}_{0}$ we will usually need $H_{1} \in \mathfrak{g}_{1}$.

EXAmple 7. For simplicity, let us calculate $\{,\}_{R}^{1}$ in the $\operatorname{PSL}(2, \mathbb{R})$ case. In this case

$$
\mathcal{K}=\left\{\left(\begin{array}{ll}
0 & 1 \\
k & 0
\end{array}\right), \quad k \text { periodic }\right\}
$$

and $\mathfrak{l}^{0}=\mathfrak{g}_{1}$. Let's choose

$$
H_{1}=\left(\begin{array}{cc}
0 & 0 \\
1 & 0
\end{array}\right)
$$

If $h: \mathcal{K} \rightarrow \mathbb{R}$ is a Hamiltonian, and $\mathbf{h}$ is its gradient, the variational derivative of any extension will be given by $H=\left(\begin{array}{cc}a & \mathbf{h} \\ b & -a\end{array}\right)$ and condition $H_{x}+[K, H] \in \mathfrak{l}^{0}$ implies $a=\frac{1}{2} \mathbf{h}_{x}$ and $b=-\frac{1}{2} \mathbf{h}_{x} x+k \mathbf{h}$. Therefore, the Poisson bracket $\{,\}_{R}^{1}$ is defined as

$$
\begin{gathered}
\{h, g\}_{R}^{1}(k)=\frac{1}{2} \int_{S^{1}} \operatorname{tr}\left(\left[H_{1}, H\right] G\right) d x \\
=\frac{1}{2} \int_{S^{1}} \operatorname{tr}\left(\begin{array}{cc}
-\mathbf{h} a_{g} & -\mathbf{h} \mathbf{g} \\
\mathbf{h} b_{g}+2 a_{h} a_{g} & 2 a_{h} \mathbf{g}-\mathbf{h} a_{g}
\end{array}\right) d x=\int_{S^{1}}\left(a_{h} \mathbf{g}-\mathbf{h} a_{g}\right) d x=\int_{S^{1}} \mathbf{h}_{x} \mathbf{g} d x .
\end{gathered}
$$

Hence, the reduced structure is $D_{x}$, which is the well-known first Hamiltonian structure for $\mathrm{KdV}$.

EXAmple 8. Consider $G=O(4,1)$. It was shown in [M3] that $\mathcal{K}$ is given by matrices of the form

$$
\left(\begin{array}{ccccc}
0 & 1 & 0 & 0 & 0 \\
\mathbf{k}_{1} & 0 & 0 & 0 & 1 \\
\mathbf{k}_{2} & 0 & 0 & \mathbf{k}_{3} & 0 \\
0 & 0 & -\mathbf{k}_{3} & 0 & 0 \\
0 & \mathbf{k}_{1} & \mathbf{k}_{2} & 0 & 0
\end{array}\right)
$$


One can check that (7.1) reduces only if

$$
H_{1}=\left(\begin{array}{lllll}
0 & 0 & 0 & 0 & 0 \\
1 & 0 & 0 & 0 & 0 \\
0 & 0 & 0 & 0 & 0 \\
0 & 0 & 0 & 0 & 0 \\
0 & 1 & 0 & 0 & 0
\end{array}\right) .
$$

In that case, if we write the reduced Poisson bracket as $\{h, g\}_{R}^{1}\left(\mathbf{k}=\int_{S^{1}} \frac{\delta g}{\delta \mathbf{k}}^{T} \mathcal{D} \frac{\delta h}{\delta \mathbf{k}} d x\right.$ for some matrix of differential operators $\mathcal{D}$ it is not hard to see that (we denote $D=\frac{d}{d x}$ )

$$
\mathcal{D}=\left(\begin{array}{ccc}
D & 0 & 0 \\
0 & -D & -\frac{\mathbf{k}_{3}}{\mathbf{k}_{2}} D \\
0 & -D \frac{\mathbf{k}_{3}}{\mathbf{k}_{2}} & D \frac{1}{\mathbf{k}_{2}} D \frac{1}{\mathbf{k}_{2}} D
\end{array}\right)
$$

Computations similar to those in [M6] show that other constant elements do not produce Poisson structures.

Example 9. Consider $G=S p(4)$. In [M6] it was shown that $\mathcal{K}$ is defined by matrices of the form

$$
\left(\begin{array}{cccc}
0 & \mathbf{k}_{1} & 1 & 0 \\
\mathbf{k}_{1} & 0 & 0 & 1 \\
\mathbf{k}_{2} & 0 & 0 & \mathbf{k}_{1} \\
0 & \mathbf{k}_{3} & -\mathbf{k}_{1} & 0
\end{array}\right)
$$

It was also shown in [M6] that no value of $H_{1}$ will produce reduced Poisson structures compatible with the geometric Poisson structure.

On the other hand, some Poisson submanifolds of $\mathcal{K}$ have been identified as producing completely integrable systems. For example, when $G=O(4,1)$ one can restrict to $\mathbf{k}_{3}=0$ to obtain first and second Hamiltonian structures for the complexly coupled $\mathrm{KdV}$ equations. If $G=S p(4)$, we can restrict to $\mathbf{k}_{1}=0$ and get a decouple system of $\mathrm{KdV}$ equations. This holds true in general for $S p(2 n)$ (see [M6]).

8. Further questions. It is hard to briefly describe all the related problems that remain open in this topic since there are many possible applications of the classical geometric machinery to several subjects. One of the most important ones is, of course, the thorough study of integrable systems attached to these brackets. Some new results have been obtained in [M6] where the author found integrable systems associated to geometric evolutions of Lagrangian planes in symplectic $\mathbb{R}^{2 n}$. These systems are biHamiltonian with respect to the brackets presented here in the case of the symplectic group. In general, one needs to classify reducible and invertible companion brackets and find integrable systems using $\{,\}_{R}$ and $\{,\}_{R}^{1}$. All best known Poisson brackets used for integration of PDEs are now known to be geometric, sometimes associated to more than one geometry as it is the case with $\mathrm{KdV}$ (appearing in projective as structures for KdV and in special affine as structures for Sawada-Kotera equations) and $\mathrm{mKdV}$ brackets (appearing in several examples).

An interesting question raised by Prof. Peter Olver is whether $\{,\}_{R}$ is related to the invariantization of Euler-Lagrange operator associated to invariant variational problems, and how. Work in that direction is under way. 
A second topic of much interest is the role of the curvature in integration. In the constant curvature Riemannian case ([MS]) it was the curvature tensor that provided one of the symplectic operators used for integration. The precise role of this tensor in the integrability picture is very intriguing, especially in the general case of a Cartan geometry, where there are several possible choices of curvature tensors (see [Sh]).

It will also be interesting to link Drinfel'd and Sokolov's brackets to the ones presented here. Drinfel'd and Sokolov obtained what they called KdV-type integrable systems associated to their bracket. They also defined their reduced manifold as defined by Lax operators. The geometric background in spaces of Lax operators (and in general with differential operators) could lead to finding normal forms for those operators, the way it was done in [M5]. This is a traditional analysis problem. Hence, it would be interesting to know how both, DS and geometric brackets are related and to study the corresponding normal forms using transversal sections. Work in that direction is also under way.

9. Appendix: The order of the differential invariants of curves in flat semisimple homogeneous spaces. This section proves a result that was used in section 5. We will show that a generic parametrized curve in a flat homogeneous semisimple space does not have differential invariants of first order (we are not counting here the parameter $x$, which has order zero).

THEOREM 11. Let $G / H$ be a flat semisimple homogeneous space. If $\mathbf{k}$ is a differential invariant for a curve $u: I \rightarrow G / H$, then the order of $\mathbf{k}$ is greater or equal to two. That is, there are no differential invariants of order one.

We have seen that the first prolonged action of the group is given by the linear isotropy faithful representation of $G_{0}$ on $\mathfrak{g}_{-1}$. Hence, to show that there are no first order differential invariants for these geometries we merely need to analyze the rank of this action and show that it has maximal rank.

The Lie algebras of flat semisimple groups are classified in [KO]. They are direct sums of simple ones found in the list below. Furthermore, each simple Lie algebra has a splitting of the form (2.1) and the adjoint action also splits accordingly ([Oc]). Hence, to prove the general case it suffices to prove that the first prolongation action has maximal rank for each simple case classified in [KO]. The following is a list of $\mathfrak{g}, \mathfrak{g}_{0}$ and $\mathfrak{g}_{-1}$ together with their isotropic representation as found in [KO]. We are denoting by $M_{p \times q}$ the space of $p$ by $q$ real matrices. All algebras are over the real numbers.

1.

$$
\begin{aligned}
\mathfrak{g} & =\operatorname{sl}(p+q), \quad \mathfrak{g}_{0}=\left\{\hat{A}=\left(\begin{array}{cc}
A & 0 \\
0 & B
\end{array}\right) \in \operatorname{sl}(p+q)\right\} \simeq \operatorname{sl}(p) \oplus \operatorname{sl}(q) \oplus \mathbb{R} \\
\mathfrak{g}_{-1} & =\left\{\hat{Y}=\left(\begin{array}{cc}
0 & Y \\
0 & 0
\end{array}\right), Y \in M_{p \times q}\right\}, \simeq M_{p \times q} \quad \ell(\hat{A})(\hat{Y})=A Y-Y B .
\end{aligned}
$$

2.

$$
\begin{aligned}
\mathfrak{g} & =\operatorname{so}(n, n) \\
\mathfrak{g}_{0} & =\left\{\hat{A}=\left(\begin{array}{cc}
A_{1} & A_{2} \\
A_{2} & A_{1}
\end{array}\right), A_{1} \in \operatorname{so}(n), A_{2} \in \operatorname{Sym}(n)\right\} \simeq\left\{A=A_{1}+A_{2}\right\} \simeq \operatorname{gl}(n) \\
\mathfrak{g}_{-1} & =\left\{\hat{Y}=\left(\begin{array}{cc}
Y & Y \\
-Y & Y
\end{array}\right), Y \in \operatorname{so}(n)\right\} \simeq \operatorname{so}(n), \quad \ell(\hat{A}) \hat{Y}=A Y+Y A^{T} .
\end{aligned}
$$


3.

$$
\begin{aligned}
\mathfrak{g}= & s o(p+1, q+1), \\
\mathfrak{g}_{0}= & \left\{A=\left(\begin{array}{cccc}
0 & 0 & a & a \\
0 & A_{1} & 0 & A_{2} \\
a & 0 & 0 & 0 \\
0 & A_{2}^{T} & 0 & A_{3}
\end{array}\right), A_{1} \in \operatorname{so}(p), A_{2} \in M_{p \times q}, A_{3} \in \operatorname{so}(q), a \in \mathbb{R}\right\} \\
\simeq & s o(p+q) \oplus \mathbb{R}, \\
\mathfrak{g}_{-1}= & \left\{\left(\begin{array}{cccc}
0 & -Y_{1}^{T} & 0 & Y_{2}^{T} \\
Y_{1} & 0 & Y_{1} & 0 \\
0 & Y_{1}^{T} & 0 & -Y_{2}^{T} \\
Y_{2} & 0 & Y_{2} & 0
\end{array}\right), Y_{1} \in M_{p \times 1}, Y_{2} \in M_{q \times 1}\right\} \simeq M_{p+q \times 1}, \\
& \ell(A) Y=a\left(\begin{array}{cc}
A_{1} & A_{2} \\
A_{2}^{T} & A_{3}
\end{array}\right)\left(\begin{array}{l}
Y_{1} \\
Y_{2}
\end{array}\right)
\end{aligned}
$$

4.

$$
\begin{aligned}
\mathfrak{g} & =\operatorname{sp}(n), \quad \mathfrak{g}_{0}=\left\{\left(\begin{array}{cc}
A & 0 \\
0 & -A^{T}
\end{array}\right), A \in \operatorname{gl}(n)\right\} \\
\mathfrak{g}_{-1} & =\left\{\left(\begin{array}{ll}
0 & Y \\
0 & 0
\end{array}\right), Y \in \operatorname{Sym}(n)\right\}, \quad \ell(A) Y=A Y+Y A^{T} .
\end{aligned}
$$

5.

$$
\mathfrak{g}=E_{6}, \quad \mathfrak{g}_{0}=s o(5,5)
$$

The representation of $\mathfrak{g}_{0}$ on $\mathfrak{g}_{-1}$ is given by the standard spin representation of $s o(5,5)$ on a 16 dimensional vector space.

6.

$$
\mathfrak{g}=E_{7}, \quad \mathfrak{g}_{0}=E_{6} \oplus \mathbb{R},
$$

and again the isotropy representation is given by the standard representation of $E_{6} \oplus \mathbb{R}$ on the space of $3 \times 3$ Cayley Hermitian matrices, as we will describe in a lemma below.

In order to finish the proof of the theorem we need to go through 1 to 6 and calculate the dimensions of generic orbits for each one of them. We will break this calculation in three lemmas, one for 1-4, the classical algebras, and one for each $E_{6}$ and $E_{7}$ cases.

LEMma 4. The minimal dimension of the isotropy algebra of the representation given in cases $1-4$ equals

$$
\operatorname{dim} \mathfrak{g}_{0}-\operatorname{dimg}_{-1}
$$

Proof. In each case we want to study the dimension of the subalgebra

$$
\left\{A \in \mathfrak{g}_{0}, \ell(A)(\xi)=0\right\}
$$

for a generic $\xi \in \mathfrak{g}_{-1}$. 
Looking into case 4 first, the dimension of elements taking $Y$ to zero coincide with the dimension of the subgroup of $\mathrm{GL}(n)$ leaving $Y$ invariant. Since $Y$ is symmetric, if generic, this will coincide with the dimension of $s o(n)$. On the other hand, $\operatorname{gl}(n)$ can be split into the direct sum of symmetric matrices and so(n), and hence (9.1) holds true.

One can reason in a parallel way in case 2. Here $Y$ is skew symmetric and the dimension of the matrices that generically leave a skew symmetric matrix invariant equals the dimension of the symplectic algebra in even dimensions, and the dimension of the algebra of matrices leaving $\left(\begin{array}{ccc}0 & 0 & 0 \\ 0 & I & 0 \\ -I & 0 & 0\end{array}\right)$ invariant in odd dimensions. In each case a simple dimension counting gives the desired result. For example, in the even case, the dimension of the symplectic algebra is $\frac{n^{2}}{2}+\frac{n}{2}$ and the dimension of $s o(n)$ is $\frac{n^{2}}{2}-\frac{n}{2}$ which adds up to the dimension of $\operatorname{gl}(n)$.

Case number 3 is simply the projective action of $s o(p, q) \oplus \mathbb{R}$ on $\mathbb{R}^{p+q}$ which is known to be transitive.

Finally, in the first case $\mathfrak{g}_{0}$ has dimension $p^{2}+q^{2}-1$ and $\mathfrak{g}_{-1}$ has dimension $p q$. Since $Y$ is a general $p \times q$ matrix, for a generic $Y$ the solution on $A$ and $B$ of equation $A Y-Y B=0$ will be given by a $p^{2}+q^{2}-1-p q$ dimensional set, using basic linear algebra.

Lemma 5. The minimal dimension of the isotropy algebra of the spin representation of $\operatorname{so}(5,5)$ is equal to $\operatorname{dim}(s o(5,5))-16=\operatorname{dimg}_{0}-\operatorname{dimg}_{-1}$.

Proof. To prove this lemma it is enough to find one element for which the dimension of the isotropy algebra is minimal and as above. That element will be generic automatically. But first let us briefly describe the spin representation of $s o(10, C)$. The complex dimension of the isotropy algebra for the complex case coincides with the real dimension of the isotropy algebra for the spin action of $s o(5,5)$, so we will study the complex case. The following description of the spin representation of $s o(2 n, C)$ is as found in $[\mathrm{F}]$. We will avoid many of the necessary details, like definitions of Clifford algebras, etc, in favor of simplicity. For a complete description of the representation please see $[\mathrm{F}]$.

Assume our standard quadratic form in $2 n$ dimensions is given by

$$
Q(v, w)=v^{T}\left(\begin{array}{cc}
0 & I \\
I & 0
\end{array}\right) w
$$

so that, if $e_{i}$ represent the standard basis in $C^{n}$, then $e_{i}$ and $e_{n+i}$ are dual elements with respect to the inner product defined by the matrix above. Assume $S O(2 n, C)$ is the group of matrices preserving this inner product. An element in $s o(2 n, C)$ is a combination of elements of the form $\alpha_{i j}=1 / 2\left(E_{i n+j}-E_{j n+i}\right)$, where we are adopting the convention of identifying $2 n$ with zero whenever $j$ or $i$ is larger than $n$.

With this description, let $W=C^{n}$ and let $\bigwedge^{*} W$ be the exterior algebra of $W$, which is $2^{n}$ dimensional. The positive spin representation, one of the irreducible representations of $s o(2 n, C)$, is defined on the $2^{n-1}$ dimensional space $V=\bigwedge^{\text {even }} W$ of even products of $W$. This space represents $\mathfrak{g}_{-1}$ in the case $\mathfrak{g}=E_{6}$. The odd powers of $W$ represent $\mathfrak{g}_{1}$ and they are the domain of the negative spin representation. First of all we will describe an action of $C^{2 n}$ on $V$ and we later use it to describe the representation of $s o(2 n, C)$. 
If $e_{i}$ is such that $i \leq n$, and $\xi \in V$, define

$$
\lambda\left(e_{i}\right)(\xi)=e_{i} \wedge \xi .
$$

If $e_{i}$ is such that $i>n$ define $\lambda\left(e_{i}\right)(\xi)=i\left(e_{i}\right)(\xi)$, where $i$ is the interior product, that is

$$
\lambda\left(e_{i}\right)\left(w_{1} \wedge w_{2} \wedge \cdots \wedge w_{r}\right)=\sum_{j=1}^{r}(-1)^{j-1} Q\left(e_{i}, w_{j}\right)\left(w_{1} \wedge \cdots \wedge \hat{w}_{j} \wedge \cdots \wedge w_{r}\right)
$$

Now, if $\alpha_{i j} \in s o(2 n, C)$ is as above, define the following action of $s o(2 n, C)$ on $\bigwedge^{e v e n} W$

$$
\rho\left(\alpha_{i j}\right)(\xi)=\frac{1}{2}\left(\lambda\left(e_{i}\right) \circ \lambda\left(e_{j}\right)-\lambda\left(e_{j}\right) \circ \lambda\left(e_{i}\right)\right)(\xi)
$$

where $\lambda$ is defined as in (9.2) and (9.3), depending on the value of the subindex, and where $\circ$ denotes composition. Representation (9.4) is the so called positive spin representation of $s o(2 n, C)$ on $\bigwedge^{e v e n} W$.

With this definition it is not very hard to show that there are elements whose isotropy algebra has minimum dimension in the case $n=5$. Indeed, consider the vector

$$
\xi=e_{1} \wedge e_{2}+e_{2} \wedge e_{3}+e_{3} \wedge e_{4}+e_{4} \wedge e_{5}+e_{5} \wedge e_{1}
$$

It is a straight forward calculation to show that $\rho(s o(10, C))(\xi)=\bigwedge^{e v e n} W$. In fact, if we consider $\alpha_{i j}$ with $1 \leq i, j \leq 5$, one can easily check that $\rho\left(\alpha_{i j}\right)(\xi)$ generates $\bigwedge^{4} W$ as $i$ and $j$ change. If $1 \leq i \leq 5$ and $5<j \leq 10$, then one can also easily check that $\rho\left(\alpha_{i j}\right)$ will generate $\bigwedge^{2} W$ as $i$ and $j$ change, and also clearly, if $5<i, j \leq 10$ their image will generate $\bigwedge^{0} W=C$. Therefore, the isotropy algebra of this element will have the dimension established by the lemma.

Lemma 6. The minimal dimension of the isotropy algebra of the natural representation of $E_{6} \oplus \mathbb{R}$ on the space of $3 \times 3$ Hermitian Cayley matrices is $\operatorname{dim} E_{6}+1-27=$ $\operatorname{dim} \mathfrak{g}_{0}-\operatorname{dimg}_{-1}$

Proof. First of all, let us describe the natural representation of $E_{6}$ in $3 \times 3$ Hermitian Cayley matrices. More details about the following description can be found in $[\mathrm{S}]$.

Let $O$ be the eight dimensional nonassociative alternative algebra of octonions (a Cayley algebra over $\mathbb{R}$, see $[\mathrm{S}]$ ). There exists an involution of the algebra that we denote by $x \rightarrow \bar{x}$ such that $\overline{x y}=\bar{y} \bar{x}, \bar{x}=x$ and such that $x+\bar{x} \in \mathbb{R}, x \bar{x}=\bar{x} x \in \mathbb{R}$, for any $x, y \in O$. Let $H_{3 \times 3}$ be the set of matrices of the form

$$
\left(\begin{array}{ccc}
\alpha & c & \bar{b} \\
\bar{c} & \beta & a \\
b & \bar{a} & \gamma
\end{array}\right)
$$

with $\alpha, \beta, \gamma \in \mathbb{R}$ and $a, b, c \in O$. As a vector space over the real numbers, $H_{3 \times 3}$ has dimension 27 . On $H_{3 \times 3}$ define the following commutative (nonassociative) product

$$
N \cdot M=\frac{1}{2}(N M+M N),
$$


where $N M$ is the nonassociative product of matrices in $H_{3 \times 3}$. With this product, $H_{3 \times 3}$ is a Jordan algebra. Let $\xi$ be an element as in (9.5) and let us write it as

$$
\xi=\alpha E_{11}+\beta E_{22}+\gamma E_{33}+A_{1}+A_{2}+A_{3}
$$

where

$$
A_{1}=\left(\begin{array}{ccc}
0 & 0 & 0 \\
0 & 0 & a \\
0 & \bar{a} & 0
\end{array}\right)=(a)_{1}, \quad A_{2}=\left(\begin{array}{ccc}
0 & 0 & \bar{b} \\
0 & 0 & 0 \\
b & 0 & 0
\end{array}\right)=(b)_{2}, \quad A_{3}=\left(\begin{array}{ccc}
0 & c & 0 \\
\bar{c} & 0 & 0 \\
0 & 0 & 0
\end{array}\right)=(c)_{3} .
$$

A very simple calculation shows

$$
\begin{aligned}
E_{i i} \cdot E_{i i} & =E_{i i}, \quad i=1,2,3, \quad E_{i i} \cdot E_{j j}=0, \quad i \neq j, \\
E_{i i} \cdot A_{i} & =0, \quad i=1,2,3, \quad E_{i i} \cdot A_{j}=\frac{1}{2} A_{j}, \quad i \neq j, \\
(a)_{i} \cdot(b)_{i} & =(a, b)\left(E_{j j}+E_{k k}\right), \quad i \neq j, k \\
(a)_{i} \cdot(b)_{j} & =(\bar{b} \bar{a})_{k}, \quad \text { for any } i, j, k \text { cyclic permutation of } 1,2,3
\end{aligned}
$$

where $(a, b)$ is the nondegenerate bilinear form on $O$ defined as $(a, b)=\frac{1}{2}(\bar{a} b+\bar{b} a)$.

Let $L_{X}: H_{3 \times 3} \rightarrow H_{3 \times 3}$ be defined by

$$
L_{X}(\xi)=X \cdot \xi
$$

The natural representation of $E_{6} \oplus \mathbb{R}$ on $H_{3 \times 3}$ identifies $E_{6} \oplus \mathbb{R}$ with the algebra

$$
\left\{L_{X}, X \in H_{3 \times 3}\right\} \cup\left\{\left[L_{X}, L_{Y}\right], X, Y \in H_{3 \times 3}\right\}
$$

where $\left[L_{X}, L_{Y}\right]=L_{X} \circ L_{Y}-L_{Y} \circ L_{X}$ represents the commutator. This algebra acts on $H_{3 \times 3}$ using (9.6) (see [S]).

As in the previous lemma, it is easy to find an element with an isotropy algebra whose dimension matches the one given in the statement of the lemma. Indeed, if

$$
\xi=E_{11}+E_{22}+E_{33}
$$

one can check, using (9.6), that

$$
L_{X}(\xi)=X, \quad\left[L_{X}, L_{Y}\right](\xi)=0
$$

for any $X, Y \in H_{3 \times 3}$. Therefore, the dimension of the isotropy algebra of $\xi$ equals the dimension of $E_{6} \oplus \mathbb{R}$ minus the dimension of $H_{3 \times 3}$, as stated.

\section{REFERENCES}

[AF] M. ANTONOwicz AND A.P. Fordy, Hamiltonian structure of nonlinear evolution equations, in "Soliton Theory: A survey of results", Manchester University Press, New York, 1990.

[Bi] L. Bianchi, Lezioni di Geomtria Differenziale, volume 1. Spoerri, 1892.

[C] E. Cartan, La Méthode du Repère Mobile, la Théorie des Groupes Continus et les Espaces Généralisés, Exposés de Géométrie 5, Hermann, Paris, 1935.

[CQ1] K-S. ChOU AND C. QU, Integrable equations arising from motions of plane curves, Phys. D, 162 (2002), pp. 9-33.

[CQ2] K-S. Chou AND C. QU, Integrable equations arising from motions of plane curves II, J. Nonlinear Sci., 13-5 (2003), pp. 487-517.

[Da] G. Darboux, Leçons sur la Géometrie des espaces de Riemann, Gauthier-Villars, 1910. 
[DS] V. G. Drinfel'D AND V. V. Sokolov, Lie algebras and equations of Korteweg- de Vries type, in "Current problems in mathematics, Vol. 24", Itogi Nauki i Tekhniki, pages 81-180. Akad. Nauk SSSR Vsesoyuz. Inst. Nauchn. i Tekhn. Inform., Moscow, 1984.

[FO1] M. Fels, P.J. Olver, Moving coframes. I. A practical algorithm, Acta Appl. Math., (1997), pp. 99-136.

[FO2] M. Fels, P.J. Olver, Moving coframes. II. Regularization and theoretical foundations, Acta Appl. Math., (1999) pp. 127-208.

[F] W. Fulton, J. Harris, Representation Theory, Graduate Texts in Mathematics, SpringerVerlag, New York, 1991.

[G] M. L. GREEN, The moving frame, differential invariants and rigidity theorems for curves in homogeneous spaces, Duke Mathematical Journal, 45 (1978), pp. 735-779.

[Ha] R. Наsimoto, A soliton on a vortex filament, J. Fluid Mechanics, 51 (1972), pp. 477-485.

[Ki] A.A. KIRILlov, Infinite dimensional groups: their representation, orbits, invariants, Proc. of International Congress of Math, Helsinki 1978.

[K] S. Kobayashi, Transformation Groups in Differential Geometry, Classics in Mathematics, Springer-Verlag, New York, 1972.

[KO] S. Kobayashi, T. Nagano, On filtered Lie Algebras and Geometric Structures I, Journal of Mathematics and Mechanics, 13 (1964), pp. 875-907.

[Li] J. Liouville, Sur l'équation aux différences partielles $\frac{d^{2} \log \lambda}{d u d v} \pm \frac{\lambda}{2 a^{2}}=0$, J. Math. Pures Appl., 18 (1853), p. 71.

[LP1] J. Langer and R. Perline, Poisson geometry of the filament equation, J. Nonlinear Sci., 1 (1991), pp. 71-93.

[LP2] J. LANGER AND R. PeRLine, Geometric Realizations of Fordy-Kulish Nonlinear Schrödinger Systems, Pacific Journal of Mathematics, 195-1 (2000), pp. 157-177.

[M1] G. MARí BefFA, Poisson brackets associated to invariant evolutions of Riemannian curves, Pacific Journal of Mathematics, 215 (2) (2004), pp. 357-380.

[M2] G. MARí BefFa, The theory of differential invariants and KdV Hamiltonian evolutions, Bull. Soc. Math. France, 127 (1999), pp. 363-391.

[M3] G. Marí Beffa, Poisson brackets associated to the conformal geometry of curves, Transactions of the AMS, 357 (2005), pp. 2799-2827.

[M4] G. MARí BefFa, Poisson Geometry of differential invariants of curves in nonsemisimple homogeneous spaces, Proceedings of the AMS, 134 (2006), pp. 779-791.

[M5] G. Marí Beffa, Transverse sections for the Second Hamiltonian KdV structure, The Journal of Geometric Analysis, 8 (1998), pp. 385-407. Also, On the Poisson geometry of the Adler-Gel'fand-Dikii brackets, The Journal of Geometric Analysis, 6-2 (1996), pp. 207-232.

[M6] G. MARí BeFFA, On completely integrable systems associated to geometric evolutions of Lagrangian planes, Proceedings of the Royal Society of Edinburg, 137A (2007), pp. 111131.

[MR] J.E. Marsden, T. Ratiu, Reduction of Poisson Manifolds, Letters in Mathematical Physics, 11 (1986), pp. 161-169.

[MS] G. Marí Beffa, J. Sanders, J.P. Wang, The theory of differential invariants and $K d V$ Hamiltonian evolutions, Journal of Nonlinear Sciences, 2002.

[O] P.J. Olver, Equivalence, Invariance and Symmetry, Cambridge University Press, Cambridge, UK, 1995

[O2] P.J. Olver, Moving frames and singularities of prolonged group actions, Selecta Math, 6 (2000), pp. 41-77.

[Oc] T. OCHIAI. Geometry associated with semisimple flat homogeneous spaces, Transactions of the AMS, 152 (1970), pp. 159-193.

[Ov] L.V. Ovsiannikov, Group Analysis of Differential Equations, Academic Press, New York (1982).

[PS] A. Pressley, G. Segal, Loop groups, Graduate Texts in Mathematics, Springer, 1997.

[S] R.D. Schafer, An introduction to Nonassociative Algebras, Dover publications, Inc, New York, 1995.

[Sh] R.W. Sharpe, Differential Geometry: Cartan's Generalization of Klein's Erlangen Program, Graduate Texts in Mathematics, Springer, 1997.

[TT] C.L. Terng And G. Thorbergsson, Completely integrable flows on Adjoint orbits, preprint, DG.math/0108154.

[TU] C.L. Terng And K. Uhlenbeck, Poisson actions and scattering theory for integrable systems, Surveys in Differential Geometry: Integrable Systems, A supplement to J. Differential Geometry, 4 (2000), pp. 315-402.

[W] E.J. Wilczynski, Projective differential geometry of curves and ruled surfaces, B.G. Teub- 
ner, Leipzig, 1906.

[YS] Y. Yasui AND N. SASAKI, Differential geometry of the vortex filament equation, J. Geom. Phys., 28 (1998), pp. 195-207. 
G. MARÍ BEFFA 